\title{
Least energy sign-changing solutions for Kirchhoff-Poisson systems
}

\section{Guoqing Chai ${ }^{1 *}$ and Weiming Liu ${ }^{1}$}

\section{"Correspondence: \\ guoqingchai@hbnu.edu.cn \\ ${ }^{1}$ College of Mathematics and \\ Statistics, Hubei Normal University, \\ Huangshi, P.R. China}

\begin{abstract}
The paper deals with the following Kirchhoff-Poisson systems:

$$
\begin{cases}-\left(1+b \int_{\mathbb{R}^{3}}|\nabla u|^{2} d x\right) \Delta u+u+k(x) \phi u+\lambda|u|^{p-2} u=h(x)|u|^{q-2} u, & x \in \mathbb{R}^{3}, \\ -\Delta \phi=k(x) u^{2}, & x \in \mathbb{R}^{3},\end{cases}
$$

where the functions $k$ and $h$ are nonnegative, $0 \leq \lambda, b ; 2 \leq p \leq 4<q<6$. Via a constraint variational method combined with a quantitative lemma, some existence results on one least energy sign-changing solution with two nodal domains to the above systems are obtained. Moreover, the convergence property of $u_{b}$ as $b \searrow 0$ is established.
\end{abstract}

Keywords: Kirchhoff-Poisson systems; Least energy sign-changing solutions; Constraint variational method; Nodal domains

\section{Introduction}

Consider the following Kirchhoff-Poisson systems:

$$
\begin{cases}-\left(1+b \int_{\mathbb{R}^{3}}|\nabla u|^{2} d x\right) \Delta u+u+k(x) \phi u+\lambda|u|^{p-2} u=h(x)|u|^{q-2} u, & x \in \mathbb{R}^{3}, \\ -\Delta \phi=k(x) u^{2}, & x \in \mathbb{R}^{3},\end{cases}
$$

where $k$ and $h$ are nonnegative functions, $0 \leq \lambda, b ; 2 \leq p \leq 4<q<6$.

When $b=0$, systems (1.1) reduce to the following Schrödinger-Poisson systems:

$$
\begin{cases}-\Delta u+u+k(x) \phi u+\lambda|u|^{p-2} u=h(x)|u|^{q-2} u, & x \in \mathbb{R}^{3}, \\ -\Delta \phi=k(x) u^{2}, & x \in \mathbb{R}^{3},\end{cases}
$$

which stem from quantum mechanics and have important applications in the semiconductor. From the physical viewpoint, the above systems have been introduced as a physical model describing a charged wave interacting with its own electrostatic field in quantum mechanics. The unknowns $u$ and $\phi$ represent the wave functions associated to the particle and electric potential. For more details, one can refer to [1-3] and the references therein.

(c) The Author(s) 2019. This article is distributed under the terms of the Creative Commons Attribution 4.0 International License (http://creativecommons.org/licenses/by/4.0/), which permits unrestricted use, distribution, and reproduction in any medium, provided you give appropriate credit to the original author(s) and the source, provide a link to the Creative Commons license, and indicate if changes were made. 
In [4], Cerami and Vaira studied the following Schrödinger-Poisson systems:

$$
\begin{cases}-\Delta u+u+k(x) \phi(x) u=a(x)|u|^{p-1} u, & x \in \mathbb{R}^{3}, \\ -\Delta \phi=k(x) u^{2}, & x \in \mathbb{R}^{3},\end{cases}
$$

with $p \in(3,5)$. Under some suitable conditions, some existence results on positive solutions were obtained. Recently, Zhong and Tang [5] investigated the following SchrödingerPoisson systems:

$$
\begin{cases}-\Delta u+u+k(x) \phi(x) u=\lambda f(x)+|u|^{4} u, & x \in \mathbb{R}^{3}, \\ -\Delta \phi=k(x) u^{2}, & x \in \mathbb{R}^{3},\end{cases}
$$

where the functions $k$ and $f$ are nonnegative, $0<\lambda<\lambda_{1}$ and $\lambda_{1}$ is the eigenvalue of the problem $-\Delta u+u=\lambda f(x) u$ in $H^{1}\left(\mathbb{R}^{3}\right)$. Via the variational method, the authors obtained the existence results on the ground state sign-changing solution for $0<\lambda<\lambda_{1}$. Replacing $u$ with $V(x) u$ in (1.3), Batista and Furtado in [6] studied the following systems:

$$
\begin{cases}-\Delta u+V(x) u+k(x) \phi u=a(x)|u|^{p-1} u, & x \in \mathbb{R}^{3}, \\ -\Delta \phi=k(x) u^{2}, & x \in \mathbb{R}^{3},\end{cases}
$$

where $p \in(3,5)$ and $a(x)$ satisfies some mild conditions, especially, the potential function $V$ can be nonconstant and indefinite in sign. By a variational approach, they also get some results of the existence of one nonnegative solution and one sign-changing solution. For the related research on this problem, the reader can refer to the literature [7-14].

On the other hand, if $k=0$ in (1.1), then (1.1) reduce to the following Kirchhoff-type problem:

$$
-\left(1+b \int_{\mathbb{R}^{3}}|\nabla u|^{2} d x\right) \Delta u+u+\lambda|u|^{p-2} u=h(x)|u|^{q-2} u, \quad x \in \mathbb{R}^{3} .
$$

Associated with the above problem, we have to mention the following Kirchhoff Dirichlet problem:

$$
\begin{cases}-\left(a+b \int_{\Omega}|\nabla u|^{2} d x\right) \Delta u=f(x, u), & x \in \Omega, \\ u=0, & x \in \partial \Omega,\end{cases}
$$

which stems from the stationary analogue of the equation

$$
\rho \frac{\partial^{2} u}{\partial t^{2}}-\left(\frac{P_{0}}{h}+\frac{E}{2 L} \int_{0}^{L}\left|\frac{\partial u}{\partial x}\right|^{2} d x\right) \frac{\partial^{2} u}{\partial x^{2}}=0
$$

proposed by Kirchhoff regarded as an extension of the classical D'Alembert wave equation on free vibrations of elastic strings. Due to its importance on the physical background, the Kirchhoff boundary problem received increasingly more attention. Recently, with the help of the variational methods, a number of results on the existence and multiplicity of 
solutions for the Kirchhoff problem

$$
\begin{cases}-\left(a+b \int_{\mathbb{R}^{N}}|\nabla u|^{2} d x\right) \Delta u=f(u), & x \in \Omega, \\ u=0, & x \in \partial \Omega,\end{cases}
$$

have been established under various suitable conditions, where $\Omega \subset \mathbb{R}^{N}$ is a bounded domain with a smooth boundary $\partial \Omega$ and $f$ satisfies various suitable conditions; see, for example [15-21] and the references therein. Recently, Baraket and Molica Bisci in [22] studied the following Kirchhoff-type problem:

$$
\begin{cases}-\left(a+b \int_{\Omega}|\Delta u|^{2}\right) \Delta u=\lambda f(x, u)+\mu g(x, u) & \text { in } \Omega, \\ u=0 & \text { on } \partial \Omega,\end{cases}
$$

where $\Omega \subset \mathbb{R}^{N}(N \geq 3)$ is a bounded open subset. Under some suitable conditions, the authors obtained multiplicity results via applying the three critical points theorem. Very recently, $\mathrm{Xu}$ and Chen in [23] investigated the following Kirchhoff-type problem:

$$
\left\{\begin{array}{l}
-\left(a+b \int_{\mathbb{R}^{3}}|\Delta u|^{2}\right) \Delta u+V u=f(u) \text { in } \mathbb{R}^{3}, \\
u \in H^{1}\left(\mathbb{R}^{3}\right),
\end{array}\right.
$$

and established the existence of a ground state solution by applying a critical point theorem similar to the mountain pass lemma (see [24]). Moreover, for the related research on fractional Kirchhoff-type and Schrödinger-type problems, the reader can refer to [25, 26] and to the references therein and the monograph [27] published recently. However, regarding the existence of sign-changing solutions for the Kirchhoff problem, there are very few results in the literature. Recently, Shuai in [28] studied the existence of the least energy sign-changing solution of problem (1.5) and its convergence property on $\left\{u_{n}\right\}$ as $b \searrow 0$. Later, under conditions different from [28], with the help of some analytical techniques and a non-Nehari manifold method, Tang and Cheng in [29] further studied problem (1.5) and obtained some existence results on a ground state sign-changing solution $u_{b}$ as well as its convergence property.

When $b \neq 0$, and $k \neq 0$, the systems (1.1) stand for Kirchhoff-Poisson systems. Because the nonlocal terms $b \int_{\mathbb{R}^{3}}\left(|\nabla u|^{2} d x\right) \Delta u$ and $\phi_{u}$ are involved in the equation, the problem is totally different from the case $b=0$ and $k=0$. In [30], Zhang considered the following general singular Kirchhoff-Poisson systems:

$$
\begin{cases}-\left(a+b \int_{\Omega}|\Delta u|^{2}\right) \Delta u+\phi u=\lambda h f(u)+g(u), & \text { in } \Omega, \\ -\Delta \phi=u^{2}, & \text { in } \Omega, \\ u>0, & \text { in } \Omega, \\ u=\phi=0, & \text { on } \partial \Omega,\end{cases}
$$

where $\Omega \subset \mathbb{R}^{3}$ is a smooth bounded domain with boundary $\partial \Omega$, constants $a>0, b \geq$ 0 and $\lambda>0$ is a parameter, functions $f, g, h$ satisfy some conditions. By combining the variational method with a perturbation method, the author obtained the existence of two 
positive solutions if the parameter $\lambda$ is small enough. In [31], Liu and Wang investigated the following Kirchhoff-Poisson systems:

$$
\begin{cases}-\left(a+b \int_{\mathbb{R}^{3}}|\Delta u|^{2}\right) \Delta u+u-q K(x) \phi u=f(x)|u|^{p-1} u, & x \in \mathbb{R}^{3}, \\ -\Delta \phi=q K(x) u^{2}, & x \in \mathbb{R}^{3},\end{cases}
$$

where the constants $a>0, b \geq 0,1<p<5, q>0$ and the functions $f, K: \mathbb{R}^{3} \rightarrow \mathbb{R}$ are nonnegative. Applying the critical point theorem with parameter $\lambda$ (see [24]), the authors obtained the existence of a positive solution as well as a ground state solution with $q=1$ corresponding to its limit problem. Moreover, very recently, Wang, Rădulescu and Zhang in [32] studied a kind of fractional Kirchhoff-Poisson systems as follows:

$$
\begin{cases}M\left([u]_{s}^{2}\right)(-\Delta)^{s} u+V(x) u+\phi(x) u=\lambda f(x, u) & \text { in } \mathbb{R}^{3}, \\ (-\Delta)^{t} \phi(x)=u^{2} & \text { in } \mathbb{R}^{3},\end{cases}
$$

where $s, t \in(0,1)$ with $2 t+4 s>3, M: \mathbb{R}_{0}^{+} \rightarrow \mathbb{R}^{+}$is a continuous function satisfying certain assumptions, the potential function $V: \mathbb{R}^{3} \rightarrow \mathbb{R}^{+}$is continuous, $f$ satisfies a Carathéodory condition, $\lambda$ is a positive parameter. By applying the fountain theorem for the subcritical case and the symmetric mountain pass theorem for the critical case, respectively, the authors obtained infinitely many solutions for the system. Different from the works mentioned above, in the present paper, we shall combine a constraint variational method with quantitative deformation properties to establish the existence results as regards one least energy sigh-changing solution with two nodal domains to problem (1.1). Moreover, we also study the convergence property on $u_{b}$ as $b \searrow 0$.

\section{Preliminaries}

Throughout this paper, we always assume the following conditions hold.

(l) $0 \leq \lambda, b ; 2 \leq p \leq 4<q<6$.

(k) $k \in L^{2}\left(\mathbb{R}^{3}\right) \cap L^{\infty}\left(\mathbb{R}^{3}\right) \backslash\{0\}$ and $k(x) \geq 0$ for a.e. $x \in \mathbb{R}^{3}$.

(h) $h(x)>0$ for a.e. $x \in \mathbb{R}^{3}$ and there exists $q_{1} \in(q, 6)$ such that $h \in L^{\frac{6}{6-q_{1}}}$.

In addition, $\mathbb{R}_{+}=[0, \infty), \mathbb{R}_{+}^{0}=(0, \infty), D^{1,2}\left(\mathbb{R}^{3}\right)$ is the Sobolev space equipped with the norm $\|u\|_{D^{1,2}}=\left(\int_{\mathbb{R}^{3}}|\nabla u|^{2} d x\right)^{\frac{1}{2}}$ and $L^{s}$ is the Lebesgue space with norm $|u|_{s}=\left(\int_{\mathbb{R}^{3}}|u|^{s} d x\right)^{\frac{1}{s}}$ for $s \geq 1$. Also, $H^{1}\left(\mathbb{R}^{3}\right)$ is the Sobolev space equipped with the norm

$$
\|u\|=\left(\int_{\mathbb{R}^{3}}\left(|\nabla u|^{2}+u^{2}\right) d x\right)^{\frac{1}{2}} .
$$

$C$ is for various positive constants, which can be different from one line to another line in the text.

Let $\bar{S}$ be the best Sobolev constant for the embedding of $D^{1,2}\left(\mathbb{R}^{3}\right)$ in $L^{6}\left(\mathbb{R}^{3}\right)$. That is,

$$
\bar{S}=\inf _{u \in D^{1,2}\left(\mathbb{R}^{3}\right) \backslash\{0\}} \frac{\|u\|_{D^{1,2}}}{|u|_{6}} .
$$

Similarly,

$$
S_{r}=\inf _{u \in H^{1}\left(\mathbb{R}^{3}\right) \backslash\{0\}} \frac{\left(\int_{\mathbb{R}^{3}}\left(|\nabla u|^{2}+u^{2}\right) d x\right)^{\frac{1}{2}}}{|u|_{r}}, \quad r \in[1,6] .
$$


For any fixed $u \in H^{1}\left(\mathbb{R}^{3}\right)$, from the Lax-Milgram theorem it follows that there exists an unique $\phi_{u} \in D^{1,2}\left(\mathbb{R}^{3}\right)$ that satisfies $-\Delta \phi=k(x) u^{2}$ weakly, that is, for any $v \in D^{1,2}\left(\mathbb{R}^{3}\right)$,

$$
\int_{\mathbb{R}^{3}} \nabla \phi_{u} \cdot \nabla v d x=\int_{\mathbb{R}^{3}} k(x) u^{2} v d x
$$

Moreover,

$$
\phi_{u}(x)=\frac{1}{4 \pi} \int_{\mathbb{R}^{3}} \frac{k(y) u^{2}(y)}{|x-y|} d y .
$$

Let

$$
L_{\phi_{u}}(v)=\int_{\mathbb{R}^{3}} k(x) \phi_{u} v^{2} d x, \quad v \in H^{1}\left(\mathbb{R}^{3}\right),
$$

then

$$
L_{\phi_{u}}(v)=\frac{1}{4 \pi} \int_{\mathbb{R}^{3}} \int_{\mathbb{R}^{3}} \frac{k(x) k(y) u^{2}(x) v^{2}(y)}{|x-y|} d x d y .
$$

Clearly, the energy functional associated with (1.1) can be expressed by

$$
\begin{aligned}
J_{b}(u)= & \frac{1}{2}\|u\|^{2}+\frac{b}{4}|\nabla u|_{2}^{4}+\frac{1}{4} \int_{\mathbb{R}^{3}} k(x) \phi_{u}(x) u^{2} d x \\
& +\frac{\lambda}{p} \int_{\mathbb{R}^{3}}|u|^{p} d x-\frac{1}{q} \int_{\mathbb{R}^{3}} h(x)|u|^{q} d x .
\end{aligned}
$$

Lemma 2.1 ([5]) Suppose that $k \in L^{\infty}\left(\mathbb{R}^{3}\right)$. Then, for any $u \in H^{1}\left(\mathbb{R}^{3}\right)$, there exists $C>0$ such that

$$
L_{\phi_{u}}(u)=\int_{\mathbb{R}^{3}} k(x) \phi_{u} u^{2} d x=\int_{\mathbb{R}^{3}}\left|\nabla \phi_{u}\right|^{2} d x \leq C\|u\|^{4} .
$$

Lemma 2.2 Assume that condition ( $k$ ) holds. Then we have

(i) $\phi_{u} \geq 0$, for any $u \in H^{1}\left(\mathbb{R}^{3}\right)$;

(ii) for any $t \in R, \phi_{t u}=t^{2} \phi_{u}$;

(iii) $\left\|\phi_{u}\right\|_{D^{1,2}} \leq \bar{S}^{-1} S_{6}^{-2}|k|_{2}\|u\|^{2}$;

(iv) $\left|\phi_{u}\right|_{6} \leq \bar{S}^{-1}\left\|\phi_{u}\right\|_{D^{1,2}}$.

Proof Under the condition (k), the conclusions (i) and (ii) directly follow from Eq. (2.3). the conclusions (iii) and (iv) directly follow from (2.4) in [4].

For $R>0$, let $\Omega_{R}=\left\{x \in \mathbb{R}^{3}:|x| \leq R\right\}, \Omega_{R}^{C}=\left\{x \in \mathbb{R}^{3}:|x|>R\right\}$. Denote

$$
u^{+}(x)=\max \{u(x), 0\}, u^{-}(x)=\min \{u(x), 0\} .
$$


Lemma 2.3 Assume that conditions $(l),(k)$ and $(h)$ hold. Then, for any $\left\{u_{n}\right\} \subset H^{1}\left(\mathbb{R}^{3}\right)$ with $u_{n}^{ \pm} \rightarrow u^{ \pm}$weakly in $H^{1}\left(\mathbb{R}^{3}\right)$ and $u_{n}^{ \pm}(x) \longrightarrow u^{ \pm}(x)$ for a.e. $x \in \mathbb{R}^{3}$, we have

(i) (a) $\int_{\mathbb{R}^{3}} k(x) \phi_{u_{n}} u_{n}^{2} d x \rightarrow \int_{\mathbb{R}^{3}} k(x) \phi_{u} u^{2} d x$,

$$
\int_{\mathbb{R}^{3}} k(x) \phi_{u_{n}^{ \pm}}\left(u_{n}^{ \pm}\right)^{2} d x \rightarrow \int_{\mathbb{R}^{3}} k(x) \phi_{u^{ \pm}}\left(u^{ \pm}\right)^{2} d x,
$$

(b) $\int_{\mathbb{R}^{3}} k(x) \phi_{u_{n}}\left(u_{n}^{ \pm}\right)^{2} d x \rightarrow \int_{\mathbb{R}^{3}} k(x) \phi_{u}\left(u^{ \pm}\right)^{2} d x$,

(c) $\int_{\mathbb{R}^{3}} k(x) \phi_{u_{n}^{+}}\left(u_{n}^{-}\right)^{2} d x \rightarrow \int_{\mathbb{R}^{3}} k(x) \phi_{u^{+}}\left(u^{-}\right)^{2} d x$,

(d) $\int_{\mathbb{R}^{3}} k(x) \phi_{u_{n}} u_{n} \varphi d x \rightarrow \int_{\mathbb{R}^{3}} k(x) \phi_{u} u \varphi d x, \quad \forall \varphi \in H^{1}\left(\mathbb{R}^{3}\right)$.

(ii) (a) $\int_{\mathbb{R}^{3}} h(x)\left|u_{n}\right|^{q} d x \rightarrow \int_{\mathbb{R}^{3}} h(x)|u|^{q} d x$,

(b) $\int_{\mathbb{R}^{3}} h(x)\left|u_{n}\right|^{q-2} u_{n} \varphi d x \rightarrow \int_{\mathbb{R}^{3}} h(x)|u|^{q-2} u \varphi d x, \quad \forall \varphi \in H^{1}\left(\mathbb{R}^{3}\right)$,

(c) $\int_{\mathbb{R}^{3}}\left|u_{n}\right|^{p-2} u_{n} \varphi d x \rightarrow \int_{\mathbb{R}^{3}}|u|^{p-2} u \varphi d x, \quad \forall \varphi \in H^{1}\left(\mathbb{R}^{3}\right)$.

Proof Item (i) Conclusions (a) and (d) follow from Lemma 6 in [5] and Lemma 2.1 in [6] and Lemma 2.1 in [4]. Conclusion (b) and (c) can be similarly proved, we omit it.

Item (ii). We only prove that $\int_{\mathbb{R}^{3}} h(x)\left|u_{n}\right|^{q} d x \rightarrow \int_{\mathbb{R}^{3}} h(x)|u|^{q} d x$. The other relations can be obtained similarly.

In fact, the condition $q<q_{1}<6$ implies that $4<\frac{6 q}{q_{1}}<6$. Let $r=\frac{6 q}{q_{1}}$. The sequence $u_{n} \rightarrow u$ weakly in $H^{1}\left(\mathbb{R}^{3}\right)$ shows that $\left\{u_{n}\right\}$ is bounded in $H^{1}\left(\mathbb{R}^{3}\right)$. Therefore, by $(2.2)$ there exists $M>0$ such that $\left|u_{n}\right|_{r}^{q} \leq M,|u|_{r}^{q} \leq M$. Thus

$$
\begin{aligned}
\left|\int_{\Omega_{R}^{C}} h(x)\left(\left|u_{n}\right|^{q}-|u|^{q}\right) d x\right| & \leq \int_{\Omega_{R}^{C}} h(x)\left(\left|u_{n}\right|^{q}+|u|^{q}\right) d x \\
& \leq\left(\int_{\Omega_{R}^{C}} h^{\frac{6}{6-q_{1}}} d x\right)^{\frac{6-q_{1}}{6}}\left(\left|u_{n}\right|_{r}^{q}+|u|_{r}^{q}\right) \\
& \leq 2 M\left(\int_{\Omega_{R}^{C}} h^{\frac{6}{6-q_{1}}} d x\right)^{\frac{6-q_{1}}{6}} .
\end{aligned}
$$

Because $h \in L_{\frac{6}{6-q_{1}}}$, we can choose $R>0$ large enough so that $2 M\left(\int_{\Omega_{R}^{C}} h^{\frac{6}{6-q_{1}}} d x\right)^{\frac{6-q_{1}}{6}}<\varepsilon$, and therefore,

$$
\left|\int_{\Omega_{R}^{C}} h(x)\left(\left|u_{n}\right|^{q}-|u|^{q}\right) d x\right|<\varepsilon
$$

On the other hand, by the absolute continuity on integral together with $h \in L_{\frac{6}{6-q_{1}}}$, for any $\eta>0$, there is $\delta>0$ such that $\left(\int_{G}|h(x)|^{\frac{6}{6-q_{1}}} d x\right)^{\frac{6-q_{1}}{6}}<\eta$ for each $G \subset \Omega_{R}$ with mes $G<\delta$. 
Then

$$
\int_{G} h(x)\left|u_{n}\right|^{q} d x \leq\left|u_{n}\right|_{r}^{q}\left(\int_{G}|h(x)|^{\frac{6}{6-q_{1}}} d x\right)^{\frac{6-q_{1}}{6}}<M \eta .
$$

So, we can apply the Vitali theorem to obtain

$$
\lim _{n \rightarrow \infty} \int_{\Omega_{R}} h(x)\left|u_{n}\right|^{q} d x=\int_{\Omega_{R}} h(x)|u|^{q} d x
$$

Hence,

$$
\begin{aligned}
\lim _{n \rightarrow \infty} \int_{\mathbb{R}^{3}} h(x)\left|u_{n}\right|^{q} d x & =\lim _{n \rightarrow \infty} \int_{\Omega_{R}^{c}} h(x)\left|u_{n}\right|^{q} d x+\lim _{n \rightarrow \infty} \int_{\Omega_{R}} h(x)\left|u_{n}\right|^{q} d x \\
& =\int_{\Omega_{R}^{c}} h(x)|u|^{q} d x+\int_{\Omega_{R}} h(x)|u|^{q} d x=\int_{\mathbb{R}^{3}} h(x)|u|^{q} d x .
\end{aligned}
$$

The proof is complete.

In terms of Lemmas 2.1-2.3, it can be verified that $J_{b}$ is well defined on $H^{1}\left(\mathbb{R}^{3}\right)$ and is of $C^{1}$ as well as

$$
\begin{aligned}
\left\langle J_{b}^{\prime}(u), v\right\rangle= & \int_{\mathbb{R}^{3}}(\nabla u \cdot \nabla v+u v) d x+b|\nabla u|_{2}^{2}\left(\int_{\mathbb{R}^{3}} \nabla u \cdot \nabla v d x\right) \\
& +\int_{\mathbb{R}^{3}} k(x) \phi_{u} u v d x+\lambda \int_{\mathbb{R}^{3}}|u|^{p-2} u v d x-\int_{\mathbb{R}^{3}} h(x)|u|^{q-2} u v d x .
\end{aligned}
$$

Obviously, $u \in H^{1}\left(\mathbb{R}^{3}\right)$ is a critical point of $J_{b}$ if and only if $\left(u, \phi_{u}\right)$ is a solution of systems (1.1). Noting that $\phi_{u}$ is nonnegative for any $u \in H^{1}\left(\mathbb{R}^{3}\right),\left(u, \phi_{u}\right)$ is a sign-changing solution of system (1.1) if and only if $u$ is a critical point of $J_{b}$ with $u^{ \pm} \neq 0$.

By (2.3)-(2.4) and the Fubini theorem, we know that

$$
L_{\phi_{u^{+}}}\left(u^{-}\right)=\int_{\mathbb{R}^{3}} k(x) \phi_{u^{+}}\left(u^{-}\right)^{2} d x=\int_{\mathbb{R}^{3}} k(x) \phi_{u^{-}}\left(u^{+}\right)^{2} d x=L_{\phi_{u^{-}}}\left(u^{+}\right)
$$

and

$$
\begin{aligned}
& J_{b}(u)=J_{b}\left(u^{+}\right)+J_{b}\left(u^{-}\right)+\frac{b}{2}\left|\nabla u^{+}\right|_{2}^{2}\left|\nabla u^{-}\right|_{2}^{2}+\frac{1}{2} L_{\phi_{u^{+}}}\left(u^{-}\right), \\
& \left\langle J_{b}^{\prime}(u), u^{+}\right\rangle=\left\langle J_{b}^{\prime}\left(u^{+}\right), u^{+}\right\rangle+b\left|\nabla u^{+}\right|_{2}^{2}\left|\nabla u^{-}\right|_{2}^{2}+L_{\phi_{u^{+}}}\left(u^{-}\right), \\
& \left\langle J_{b}^{\prime}(u), u^{-}\right\rangle=\left\langle J_{b}^{\prime}\left(u^{-}\right), u^{-}\right\rangle+b\left|\nabla u^{+}\right|_{2}^{2}\left|\nabla u^{-}\right|_{2}^{2}+L_{\phi_{u^{+}}}\left(u^{-}\right) .
\end{aligned}
$$

Let

$$
M_{b}=\left\{u \in H^{1}\left(\mathbb{R}^{3}\right): u^{ \pm} \neq 0,\left\langle J_{b}^{\prime}(u), u^{+}\right\rangle=\left\langle J_{b}^{\prime}(u), u^{-}\right\rangle=0\right\} .
$$

In the following, we will look for the minimum point of the functional $J_{b}$ on $M_{b}$, which is the sigh-changing solution of systems (1.1).

The following lemma is crucial and plays an important role in obtaining our main results later. 
Lemma 2.4 Assume $u \in H^{1}\left(\mathbb{R}^{3}\right)$ with $u^{ \pm} \neq 0$. Then there is an unique pair $\left(s_{u}, t_{u}\right) \in \mathbb{R}_{+}^{0} \times$ $\mathbb{R}_{+}^{0}$ satisfying that $s_{u} u^{+}+t_{u} u^{-} \in M_{b}$.

Proof For any $u \in H^{1}\left(\mathbb{R}^{3}\right)$ with $u^{ \pm} \neq 0$, clearly, by (2.6)-(2.10) we know that $s u^{+}+t u^{-} \in M_{b}$ with $(s, t) \in \mathbb{R}_{+}^{0} \times \mathbb{R}_{+}^{0}$ if and only if the pair $(s, t)$ satisfies the following systems:

$$
\left\{\begin{array}{c}
s^{2}\left\|u^{+}\right\|^{2}+b s^{4}\left|\nabla u^{+}\right|_{2}^{4}+s^{4} \int_{\mathbb{R}^{3}} k(x) \phi_{u^{+}}\left(u^{+}\right)^{2} d x+\lambda s^{p}\left|u^{+}\right|_{p}^{p} \\
\quad+s^{2} t^{2}\left[b\left|\nabla u^{+}\right|_{2}^{2}\left|\nabla u^{-}\right|_{2}^{2}+\int_{\mathbb{R}^{3}} k(x) \phi_{u^{+}}\left(u^{-}\right)^{2} d x\right]-s^{q} \int_{\mathbb{R}^{3}} h(x)\left|u^{+}\right|^{q} d x=0 \\
t^{2}\left\|u^{-}\right\|^{2}+b t^{4}\left|\nabla u^{-}\right|_{2}^{4}+t^{4} \int_{\mathbb{R}^{3}} k(x) \phi_{u^{-}}\left(u^{-}\right)^{2} d x+\lambda t^{p}\left|u^{-}\right|_{p}^{p} \\
\quad+s^{2} t^{2}\left[b\left|\nabla u^{+}\right|_{2}^{2}\left|\nabla u^{-}\right|_{2}^{2}+\int_{\mathbb{R}^{3}} k(x) \phi_{u^{+}}\left(u^{-}\right)^{2} d x\right]-t^{q} \int_{\mathbb{R}^{3}} h(x)\left|u^{-}\right|^{q} d x=0 .
\end{array}\right.
$$

To study the solvability of systems (2.11), we investigate the following auxiliary systems with a parameter $\eta \in[0,1]$ :

$$
\left\{\begin{array}{c}
s^{2}\left\|u^{+}\right\|^{2}+b s^{4}\left|\nabla u^{+}\right|_{2}^{4}+s^{4} \int_{\mathbb{R}^{3}} k(x) \phi_{u^{+}}\left(u^{+}\right)^{2} d x+\lambda s^{p}\left|u^{+}\right|_{p}^{p} \\
\quad+\eta s^{2} t^{2}\left[b\left|\nabla u^{+}\right|_{2}^{2}\left|\nabla u^{-}\right|_{2}^{2}+\int_{\mathbb{R}^{3}} k(x) \phi_{u^{+}}\left(u^{-}\right)^{2} d x\right]-s^{q} \int_{\mathbb{R}^{3}} h(x)\left|u^{+}\right|^{q} d x=0, \\
t^{2}\left\|u^{-}\right\|^{2}+b t^{4}\left|\nabla u^{-}\right|_{2}^{4}+t^{4} \int_{\mathbb{R}^{3}} k(x) \phi_{u^{-}}\left(u^{-}\right)^{2} d x+\lambda t^{p}\left|u^{-}\right|_{p}^{p} \\
\quad+\eta s^{2} t^{2}\left[b\left|\nabla u^{+}\right|_{2}^{2}\left|\nabla u^{-}\right|_{2}^{2}+\int_{\mathbb{R}^{3}} k(x) \phi_{u^{+}}\left(u^{-}\right)^{2} d x\right]-t^{q} \int_{\mathbb{R}^{3}} h(x)\left|u^{-}\right|^{q} d x=0 .
\end{array}\right.
$$

Let

$$
E=\left\{\eta \mid 0 \leq \eta \leq 1 \text { such that systems (2.12) have an unique solution in } \mathbb{R}_{+}^{0} \times \mathbb{R}_{+}^{0}\right\} .
$$

Put

$$
\left\{\begin{aligned}
\varphi_{\eta}(s, t)= & s^{2}\left\|u^{+}\right\|^{2}+b s^{4}\left|\nabla u^{+}\right|_{2}^{4}+s^{4} \int_{\mathbb{R}^{3}} k(x) \phi_{u^{+}}\left(u^{+}\right)^{2} d x+\lambda s^{p}\left|u^{+}\right|_{p}^{p} \\
& +\eta s^{2} t^{2}\left[b\left|\nabla u^{+}\right|_{2}^{2}\left|\nabla u^{-}\right|_{2}^{2}+\int_{\mathbb{R}^{3}} k(x) \phi_{u^{+}}\left(u^{-}\right)^{2} d x\right]-s^{q} \int_{\mathbb{R}^{3}} h(x)\left|u^{+}\right|^{q} d x, \\
\psi_{\eta}(s, t)= & t^{2}\left\|u^{-}\right\|^{2}+b t^{4}\left|\nabla u^{-}\right|_{2}^{4}+t^{4} \int_{\mathbb{R}^{3}} k(x) \phi_{u^{-}}\left(u^{-}\right)^{2} d x+\lambda t^{p}\left|u^{-}\right|_{p}^{p} \\
& +\eta s^{2} t^{2}\left[b\left|\nabla u^{+}\right|_{2}^{2}\left|\nabla u^{-}\right|_{2}^{2}+\int_{\mathbb{R}^{3}} k(x) \phi_{u^{+}}\left(u^{-}\right)^{2} d x\right]-t^{q} \int_{\mathbb{R}^{3}} h(x)\left|u^{-}\right|^{q} d x .
\end{aligned}\right.
$$

(1) In this part, we show that $0 \in E$.

Since $\varphi_{0}(s, t)=\varphi_{0}(s, 0), \psi_{0}(s, t)=\psi_{0}(0, t)$, the solvability on $\varphi_{0}(s, t)=0$ and $\psi_{0}(s, t)=0$ is the same. So, we only show that there an unique $\bar{t} \in \mathbb{R}_{+}^{0}$ such that $\psi_{0}(0, \bar{t})=0$.

In fact, owing to the fact that $2 \leq p \leq 4<q<6, \lambda \geq 0$, and $h(x)>0$, a.e. $x \in \mathbb{R}$, we know that $\psi_{0}(0, t)>0$ as $t>0$ is small enough and $\psi_{0}(0, t)<0$ as $t>0$ is large enough. Hence, there is a $\bar{t} \in \mathbb{R}_{+}^{0}$ such that $\psi_{0}(0, \bar{t})=0$.

Now, we prove that such a number $\bar{t} \in \mathbb{R}_{+}^{0}$ is unique. To this end, let $g(t)=t^{-2} \psi_{0}(0, t)$, $t \geq 0$.

By

$$
\begin{aligned}
g(t)= & \left\|u^{-}\right\|^{2}+b t^{2}\left|\nabla u^{-}\right|_{2}^{4}+t^{2} \int_{\mathbb{R}^{3}} k(x) \phi_{u^{-}}\left(u^{-}\right)^{2} d x+\lambda t^{p-2}\left|u^{-}\right|_{p}^{p} \\
& -t^{q-2} \int_{\mathbb{R}^{3}} h(x)\left|u^{-}\right|^{q} d x, \quad t \geq 0,
\end{aligned}
$$


we have $g(0)>0$ and $g(\bar{t})=0$. If there exists another number $t_{0}>0$ with $t_{0} \neq \bar{t}$ such that $\psi_{0}\left(0, t_{0}\right)=0$, that is, $g\left(t_{0}\right)=0$, then we will obtain a contradiction via the following argument:

Case 1. If $t_{0}<\bar{t}$, then

$$
\begin{aligned}
t_{0} g^{\prime}\left(t_{0}\right)= & 2 b t_{0}^{2}\left|u^{-}\right|_{2}^{4}+2 t_{0}^{2} \int_{\mathbb{R}^{3}} k(x) \phi_{u^{-}}\left(u^{-}\right)^{2} d x+\lambda(p-2) t_{0}^{p-2}\left|u^{-}\right|_{p}^{p} \\
& -(q-2) t_{0}^{q-2} \int_{\mathbb{R}^{3}} h(x)\left|u^{-}\right|^{q} d x
\end{aligned}
$$

On the other hand, the fact that $g\left(t_{0}\right)=0$ implies that

$$
\begin{aligned}
& b t_{0}^{2}\left|\nabla u^{-}\right|_{2}^{4}+t_{0}^{2} \int_{\mathbb{R}^{3}} k(x) \phi_{u^{-}}\left(u^{-}\right)^{2} d x \\
& \quad=-\left\|u^{-}\right\|^{2}-\lambda t_{0}^{p-2}\left|u^{-}\right|_{p}^{p}+t_{0}^{q-2} \int_{\mathbb{R}^{3}} h(x)\left|u^{-}\right|^{q} d x .
\end{aligned}
$$

Thus, substituting (2.15) into (2.14) and taking into account that $0<t_{0}, u^{-} \neq 0,2 \leq p \leq 4<$ $q<6, \lambda \geq 0$ and $h(x)>0$, a.e. $x \in \mathbb{R}^{3}$, we get

$$
t_{0} g^{\prime}\left(t_{0}\right)=(p-4) \lambda t_{0}^{p-2}\left|u^{-}\right|_{p}^{p}+(4-q) t_{0}^{q-2} \int_{\mathbb{R}^{3}} h(x)\left|u^{-}\right|^{q} d x-2\left\|u^{-}\right\|^{2}<0,
$$

which together with $g\left(t_{0}\right)=0$ implies that $g\left(t_{0}+\delta\right)<0$ as $0<\delta$ is small enough. Hence, there is a $t_{*} \in\left(t_{0}, \bar{t}\right)$ satisfying

$$
g\left(t_{*}\right)=\min _{t \in\left[t_{0}, \bar{t}\right]} g(t)<0, \quad g^{\prime \prime}\left(t_{*}\right) \geq 0 .
$$

However, observing that

$$
\begin{aligned}
t_{*}^{2} g^{\prime \prime}\left(t_{*}\right)= & 2 b t_{*}^{2}\left|\nabla u^{-}\right|_{2}^{4}+2 t_{*}^{2} \int_{\mathbb{R}^{3}} k(x) \phi_{u^{-}}\left(u^{-}\right)^{2} d x+\lambda(p-2)(p-3) t_{*}^{p-2}\left|u^{-}\right|_{p}^{p} \\
& -(q-2)(q-3) t_{*}^{q-2} \int_{\mathbb{R}^{3}} h(x)\left|u^{-}\right|^{q} d x
\end{aligned}
$$

and

$$
b t_{*}^{2}\left|\nabla u^{-}\right|_{2}^{4}+t_{*}^{2} \int_{\mathbb{R}^{3}} k(x) \phi_{u^{-}}\left(u^{-}\right)^{2} d x<-\lambda t_{*}^{p-2}\left|u^{-}\right|_{p}^{p}+t_{*}^{q-2} \int_{\mathbb{R}^{3}} h(x)\left|u^{-}\right|^{q} d x-\left\|u^{-}\right\|^{2}
$$

(following from $g\left(t_{*}\right)<0$ ), we have

$$
t_{*}^{2} g^{\prime \prime}\left(t_{*}\right)<\lambda(p-1)(p-4) t_{*}^{p-2}\left|u^{-}\right|_{p}^{p}-(q-1)(q-4) t_{*}^{q-2} \int_{\mathbb{R}^{3}} h(x)\left|u^{-}\right|^{q} d x-2\left\|u^{-}\right\|^{2}<0
$$

(noting that $2 \leq p \leq 4<q<6, \lambda \geq 0$ and $h(x)>0$, a.e. $x \in \mathbb{R}^{3}$ ), which contradicts the fact that $g^{\prime \prime}\left(t_{*}\right) \geq 0$.

Case 2 . If $\bar{t}<t_{0}$, the proof is the same. In fact, by only replacing $t_{0}$ with $\bar{t}$ in the above argument on case 1 , we also obtain a contradiction.

Hence, we have proved that $0 \in E$.

(2) In this part, we show that the set $E$ is open and closed in $[0,1]$. 
(i) $E$ is an open set in $[0,1]$.

For any fixed $\eta_{0} \in E$ and $\left(s_{0}, t_{0}\right) \in \mathbb{R}_{+}^{0} \times \mathbb{R}_{+}^{0}$ is an unique solution of (2.12) associated with $\eta=\eta_{0}$. By calculation, from (2.13) we know that

$$
\begin{aligned}
\left.\frac{\partial \varphi_{\eta_{0}}}{\partial s}\right|_{\left(s_{0}, t_{0}\right)}= & 2 s_{0} \|\left. u^{+}\right|^{2}+4 b s_{0}^{3}\left|\nabla u^{+}\right|_{2}^{4}+4 s_{0}^{3} \int_{\mathbb{R}^{3}} k(x) \phi_{u^{+}}\left(u^{+}\right)^{2} d x \\
& +2 \eta_{0} s_{0} t_{0}^{2}\left[b\left|\nabla u^{+}\right|_{2}^{2}\left|\nabla u^{-}\right|_{2}^{2}+\int_{\mathbb{R}^{3}} k(x) \phi_{u^{+}}\left(u^{-}\right)^{2} d x\right] \\
& +\lambda p s_{0}^{p-1}\left|u^{+}\right|_{p}^{p}-q s_{0}^{q-1} \int_{\mathbb{R}^{3}} h(x)\left|u^{+}\right|^{q} d x, \\
\left.\frac{\left.\partial \varphi_{\eta_{0}}\right|_{\left(s_{0}, t_{0}\right)}=}{\partial \psi_{\eta_{0}}}\right|_{\left(s_{0}, t_{0}\right)}= & 2 \eta_{0} s_{0}^{2} t_{0}\left[b \mid \nabla t_{0}^{2}\left[b\left|\nabla u^{+}\right|_{2}^{2}\left|\nabla u^{-}\right|_{2}^{2}+\int_{\mathbb{R}^{3}} k(x){\phi_{u}}_{u^{+}}\left(u^{-}\right)^{2} d x\right],\right. \\
\left.\frac{\partial \psi_{\eta_{0}}}{\partial t}\right|_{\left(s_{0}, t_{0}\right)}= & \left.2 t_{0}|| u_{\mathbb{R}^{3}} k(x) \phi_{u^{+}}\left(u^{-}\right)^{2} d x\right], \\
& +2 \eta_{0} s_{0}^{2} t_{0}\left[\left.b\left|\nabla t_{0}^{3}\right| \nabla u^{-}\right|_{2} ^{4}+4 t_{0}^{3} \int_{\mathbb{R}^{3}} k(x) \phi_{u^{-}}\left(u^{-}\right)^{2} d x\right. \\
& \left.+\lambda p u_{0}^{p-1}\left|u^{-}\right|_{2}^{p}-q \int_{\mathbb{R}^{3}}^{q-1} \int_{\mathbb{R}^{3}} h(x) \phi_{u^{+}}\left(u^{-}\right)^{2} d x\right]
\end{aligned}
$$

Again, by $\varphi_{\eta_{0}}\left(s_{0}, t_{0}\right)=0$, from (2.13) we have

$$
\begin{aligned}
b s_{0}^{3}\left|\nabla u^{+}\right|_{2}^{4}+s_{0}^{3} \int_{\mathbb{R}^{3}} k(x) \phi_{u^{+}}\left(u^{+}\right)^{2} d x \\
=-\eta_{0} s_{0} t_{0}^{2}\left[b\left|\nabla u^{+}\right|_{2}^{2}\left|\nabla u^{-}\right|_{2}^{2}+\int_{\mathbb{R}^{3}} k(x) \phi_{u^{+}}\left(u^{-}\right)^{2} d x\right] \\
-\lambda s_{0}^{p-1}\left|u^{+}\right|_{p}^{p}+s_{0}^{q-1} \int_{\mathbb{R}^{3}} h(x)\left|u^{+}\right|^{q} d x-s_{0}\left\|u^{+}\right\|^{2} .
\end{aligned}
$$

By (2.16) combined with (2.20), we get

$$
\begin{aligned}
\left.\frac{\partial \varphi_{\eta_{0}}}{\partial s}\right|_{\left(s_{0}, t_{0}\right)}= & -2 s_{0}\left\|u^{+}\right\|^{2}-2 \eta_{0} s_{0} t_{0}^{2}\left[b\left|\nabla u^{+}\right|_{2}^{2}\left|\nabla u^{-}\right|_{2}^{2}+\int_{\mathbb{R}^{3}} k(x) \phi_{u^{+}}\left(u^{-}\right)^{2} d x\right] \\
& -\lambda(4-p) s_{0}^{p-1}\left|u^{+}\right|_{p}^{p}-(q-4) s_{0}^{q-1} \int_{\mathbb{R}^{3}} h(x)\left|u^{+}\right|^{q} d x .
\end{aligned}
$$

Similarly, by $\psi_{\eta_{0}}\left(s_{0}, t_{0}\right)=0$, we can deduce that

$$
\begin{aligned}
\left.\frac{\partial \psi_{\eta_{0}}}{\partial t}\right|_{\left(s_{0}, t_{0}\right)}= & -2 t_{0}\left\|u^{-}\right\|^{2}-2 \eta_{0} s_{0}^{2} t_{0}\left[b\left|\nabla u^{+}\right|_{2}^{2}\left|\nabla u^{-}\right|_{2}^{2}+\int_{\mathbb{R}^{3}} k(x) \phi_{u^{+}}\left(u^{-}\right)^{2} d x\right] \\
& -\lambda(4-p) t_{0}^{p-1}\left|u^{-}\right|_{p}^{p}-(q-4) t_{0}^{q-1} \int_{\mathbb{R}^{3}} h(x)\left|u^{-}\right|^{q} d x .
\end{aligned}
$$


Thus, by (2.17)-(2.18) and (2.21)-(2.22) as well as $\phi_{u^{+}}, \phi_{u^{-}} \geq 0, \lambda \geq 0,2 \leq p \leq 4<q, h(x)>$ 0 , a.e. $x \in \mathbb{R}^{3}$, we have the determinant

$$
\begin{aligned}
M & =\frac{\partial \varphi_{\eta_{0}}\left(s_{0}, t_{0}\right)}{\partial s} \cdot \frac{\partial \psi_{\eta_{0}}\left(s_{0}, t_{0}\right)}{\partial t}-\frac{\partial \varphi_{\eta_{0}}\left(s_{0}, t_{0}\right)}{\partial t} \cdot \frac{\partial \psi_{\eta_{0}}\left(s_{0}, t_{0}\right)}{\partial s} \\
& >A B-4 \eta_{0}^{2} s_{0}^{3} t_{0}^{3}\left[b\left|\nabla u^{+}\right|_{2}^{2}\left|\nabla u^{-}\right|_{2}^{2}+\int_{\mathbb{R}^{3}} k(x) \phi_{u^{+}}\left(u^{-}\right)^{2} d x\right]^{2}>0,
\end{aligned}
$$

where

$$
\begin{aligned}
& A=2 s_{0}\left\|u^{+}\right\|^{2}+2 \eta_{0} s_{0} t_{0}^{2}\left(b\left|\nabla u^{+}\right|_{2}^{2}\left|\nabla u^{-}\right|_{2}^{2}+\int_{\mathbb{R}^{3}} k(x) \phi_{u^{+}}\left(u^{-}\right)^{2} d x\right), \\
& B=2 t_{0}\left\|u^{-}\right\|^{2}+2 \eta_{0} s_{0}^{2} t_{0}\left(b\left|\nabla u^{+}\right|_{2}^{2}\left|\nabla u^{-}\right|_{2}^{2}+\int_{\mathbb{R}^{3}} k(x) \phi_{u^{+}}\left(u^{-}\right)^{2} d x\right), \\
& M=\left|\begin{array}{ll}
\frac{\partial \varphi_{\eta_{0}}\left(s_{0}, t_{0}\right)}{\partial s} & \frac{\partial \varphi_{\eta_{0}}\left(s_{0}, t_{0}\right)}{\partial t} \\
\frac{\partial \psi_{\eta_{0}}\left(s_{0}, t_{0}\right)}{\partial s} & \frac{\partial \psi_{\eta_{0}}\left(s_{0}, t_{0}\right)}{\partial t}
\end{array}\right| .
\end{aligned}
$$

Hence, by the implicit function theorem, there exist an open neighborhood $V_{0}$ of $\eta_{0}$ and $\wedge_{0} \subset \mathbb{R}_{+}^{0} \times \mathbb{R}_{+}^{0}$ of $\left(s_{0}, t_{0}\right)$ such that the implicit function $s=s(\eta), t=t(\eta)$ satisfies system (2.12) on $V_{0} \times \wedge_{0}$.

Now, we show that, for any $\eta \in V_{0}$, the system (2.12) has no solution in $\left(\mathbb{R}_{+}^{0} \times \mathbb{R}_{+}^{0}\right) \backslash \wedge_{0}$.

Suppose by contradiction that there exists $\eta_{1} \in V_{0}$ such that system (2.12) has another solution $(\bar{s}, \bar{t})$ in $\left(\mathbb{R}_{+}^{0} \times \mathbb{R}_{+}^{0}\right) \backslash \wedge_{0}$ associated with $\eta_{1}$ apart from the solution $(s, t)$ in $\wedge_{0}$ associated with $\eta_{1}$. Then, by the implicit function theorem again, we can find a solution function $\bar{s}=\bar{s}(\eta), \bar{t}=\bar{t}(\eta)$ in $\left(\eta_{1}-\varepsilon, \eta_{1}+\varepsilon\right)$ for some $\varepsilon>0$, which satisfies (2.12) and goes through $\left(\eta_{1},(\bar{s}, \bar{t})\right)$.

1. If $\eta_{0}<\eta_{1}$, then consider the saturated solution $\bar{s}=\bar{s}(\eta), \bar{t}=\bar{t}(\eta)$ on its saturated interval. Since it cannot be defined at $\eta_{0}$ and cannot enter $V_{0} \times \wedge_{0}$, there exists a point $\eta_{2} \in\left[\eta_{0}, \eta_{1}\right)$ such that the solution $\bar{s}=\bar{s}(\eta), \bar{t}=\bar{t}(\eta)$ in $\left(\eta_{2}, \eta_{1}\right)$ and $\bar{s}^{2}(\eta)+\bar{t}^{2}(\eta) \rightarrow \infty$ as $\eta \rightarrow \eta_{2}^{+}$, which contradicts systems (2.12) noting that $2 \leq p \leq 4<q<6, \lambda \geq 0$, and $h(x)>0$, a.e. $x \in \mathbb{R}^{3}$. Hence $V_{0} \subset E$.

2. If $\eta_{0}>\eta_{1}$, the proof is similar.

(ii) $E$ is a closed set in $[0,1]$.

In fact, let $\left\{\eta_{n}\right\} \subset E$ be a sequence with $\eta_{n} \rightarrow \eta_{0} \in[0,1]$ and $\left(s_{n}, t_{n}\right) \in \mathbb{R}_{+}^{0} \times \mathbb{R}_{+}^{0}$ be the unique solution of (2.12) associated with $\eta_{n}$. Because the sequence $\left\{\eta_{n}\right\}$ is bounded, from (2.12) it follows that $\left\{\left(s_{n}, t_{n}\right)\right\}$ is bounded. Therefore, there exists a subsequence of $\left\{\left(s_{n}, t_{n}\right)\right\}$, still denoted by $\left\{\left(s_{n}, t_{n}\right)\right\}$, such that $\left(s_{n}, t_{n}\right) \rightarrow\left(s_{0}, t_{0}\right)$. Of course, $\left(s_{0}, t_{0}\right)$ satisfies systems $(2.12)$ for $\eta=\eta_{0}$. Furthermore, by (2.12), we have

$$
\left\|u^{+}\right\|^{2} \leq s_{n}^{q-2} \int_{\mathbb{R}^{3}} h(x)\left|u^{+}\right|^{q} d x
$$

which implies that $s_{n} \geq c_{1}>0$ for some $c_{1}>0$ because $2 \leq p \leq 4<q<6, h(x)>0$, a.e. $x \in \mathbb{R}^{3}$ and $u^{+} \neq 0$. Similarly, there exists $c_{2}>0$ such that $t_{n} \geq c_{2}>0$. Thus, $\left(s_{0}, t_{0}\right) \in \mathbb{R}_{+}^{0} \times \mathbb{R}_{+}^{0}$ is a solution of (2.12). Also, the implicit function theorem ensures that $\left(s_{0}, t_{0}\right)$ is the unique solution of (2.12) for $\eta=\eta_{0}$ again. Hence, $E$ is closed in $[0,1]$. 
Summing up the above arguments (i) and (ii), we get $E=[0,1]$, and therefore, the conclusion of Lemma 2.4 is true.

Lemma 2.5 Assume that $u \in H^{1}\left(\mathbb{R}^{3}\right)$ with $u^{ \pm} \neq 0$ and $\varphi_{1}(1,1) \leq 0, \psi_{1}(1,1) \leq 0$, where $\varphi_{1}, \psi_{1}$ are given as in (2.13) with $\eta=1$. Then the unique pair $\left(s_{u}, t_{u}\right) \in \mathbb{R}_{+}^{0} \times \mathbb{R}_{+}^{0}$ given in Lemma 2.4 satisfies $0<s_{u}, t_{u} \leq 1$.

Proof If $s_{u} \geq t_{u}>0$, then, by $s_{u} u^{+}+t_{u} u^{-} \in M_{b}$ together with (2.11), we have

$$
\begin{aligned}
s_{u}^{2}\left\|u^{+}\right\|^{2} & +s^{4} b\left|\nabla u^{+}\right|_{2}^{4}+s_{u}^{4} \int_{\mathbb{R}^{3}} k(x) \phi_{u^{+}}\left(u^{+}\right)^{2} d x \\
& +s_{u}^{4}\left[b\left|\nabla u^{+}\right|_{2}^{2}\left|\nabla u^{-}\right|_{2}^{2}+\int_{\mathbb{R}^{3}} k(x) \phi_{u^{+}}\left(u^{-}\right)^{2} d x\right] \\
\geq & -\lambda s_{u}^{p}\left|u^{+}\right|_{p}^{p}+s_{u}^{q} \int_{\mathbb{R}^{3}} h(x)\left|u^{+}\right|^{q} d x .
\end{aligned}
$$

Thus

$$
\begin{aligned}
& s_{u}^{-2}\left\|u^{+}\right\|^{2}+b\left|\nabla u^{+}\right|_{2}^{4}+\int_{\mathbb{R}^{3}} k(x) \phi_{u^{+}}\left(u^{+}\right)^{2} d x+\left[b\left|\nabla u^{+}\right|_{2}^{2}\left|\nabla u^{-}\right|_{2}^{2}+\int_{\mathbb{R}^{3}} k(x) \phi_{u^{+}}\left(u^{-}\right)^{2} d x\right] \\
& \geq-\lambda s_{u}^{p-4}\left|u^{+}\right|_{p}^{p}+s_{u}^{q-4} \int_{\mathbb{R}^{3}} h(x)\left|u^{+}\right|^{q} d x .
\end{aligned}
$$

On the other hand, the assumption $\varphi_{1}(1,1) \leq 0$ implies that

$$
\begin{aligned}
& \left\|u^{+}\right\|^{2}+b\left|\nabla u^{+}\right|_{2}^{4}+\int_{\mathbb{R}^{3}} k(x) \phi_{u^{+}}\left(u^{+}\right)^{2} d x+\left[b\left|\nabla u^{+}\right|_{2}^{2}\left|\nabla u^{-}\right|_{2}^{2}+\int_{\mathbb{R}^{3}} k(x) \phi_{u^{+}}\left(u^{-}\right)^{2} d x\right] \\
& \leq-\lambda\left|u^{+}\right|_{p}^{p}+\int_{\mathbb{R}^{3}} h(x)\left|u^{+}\right|^{q} d x .
\end{aligned}
$$

By (2.23)-(2.24), we have

$$
\left(s_{u}^{-2}-1\right)\left\|u^{+}\right\|^{2}+\lambda\left(s_{u}^{p-4}-1\right)\left|u^{+}\right|_{p}^{p} \geq\left(s_{u}^{q-4}-1\right) \int_{\mathbb{R}^{3}} h(x)\left|u^{+}\right|^{q} d x .
$$

Because $u^{+} \neq 0,0 \leq \lambda, 2 \leq p \leq 4<q<6$ and $h(x)>0$, for a.e. $x \in \mathbb{R}^{3}$, the above inequality implies that $0<s_{u} \leq 1$, and so, $0<t_{u} \leq 1$.

For the case $0<t_{u} \leq s_{u}$, the proof is similar.

Let $m_{b}:=\inf \left\{J_{b}(u) \mid u \in M_{b}\right\}$. We have the following lemma.

Lemma 2.6 Assume that $(l),(k),(h)$ hold. Then $m_{b}>0$ can be achieved at some point $u_{b} \in M_{b}$.

Proof For any $u \in M_{b}$, by $\left\langle J_{b}^{\prime}(u), u\right\rangle=0$ and (2.2), we have

$$
\begin{aligned}
\|u\|^{2} & \leq\|u\|^{2}+b|\nabla u|_{2}^{4}+\int_{\mathbb{R}^{3}} k(x) \phi_{u} u^{2} d x+\lambda \int_{\mathbb{R}^{3}}|u|^{p} d x \\
& =\int_{\mathbb{R}^{3}} h(x)|u|^{q} d x \leq|h|_{\frac{6}{6-q_{1}}}|u|_{r}^{q} \leq|h|_{\frac{6}{6-q_{1}}} S_{r}^{-q}\|u\|^{q},
\end{aligned}
$$


where $r=\frac{6 q}{q_{1}}$ satisfying $4<r<6$. Hence, $\|u\| \geq c_{0}>0$, where $c_{0}=\left(\frac{S_{r}^{q}}{|h| \frac{6}{6-q_{1}}}\right)^{\frac{1}{q-2}}$. Also, by

$$
\begin{aligned}
J_{b}(u)= & J_{b}(u)-\frac{1}{q}\left\langle J_{b}^{\prime}(u), u\right\rangle \\
= & \left(\frac{1}{2}-\frac{1}{q}\right)\|u\|^{2}+\left(\frac{1}{4}-\frac{1}{q}\right) b|\nabla u|_{2}^{4}+\left(\frac{1}{4}-\frac{1}{q}\right) \int_{\mathbb{R}^{3}} k(x) \phi_{u} u^{2} d x \\
& +\lambda\left(\frac{1}{p}-\frac{1}{q}\right)|u|_{p}^{p} \\
\geq & \left(\frac{1}{2}-\frac{1}{q}\right)\|u\|^{2} \geq\left(\frac{1}{2}-\frac{1}{q}\right) c_{0}^{2}>0,
\end{aligned}
$$

we have $m_{b} \geq\left(\frac{1}{2}-\frac{1}{q}\right) c_{0}^{2}>0$.

Let $\left\{u_{n}\right\} \subset M_{b}$ with $J_{b}\left(u_{n}\right) \rightarrow m_{b}$ as $n \rightarrow \infty$. By

$$
J_{b}\left(u_{n}\right)=J_{b}\left(u_{n}\right)-\frac{1}{q}\left\langle J_{b}^{\prime}\left(u_{n}\right), u_{n}\right\rangle \geq\left(\frac{1}{2}-\frac{1}{q}\right)\left\|u_{n}\right\|^{2},
$$

and $J_{b}\left(u_{n}\right) \rightarrow m_{b}$, we know that $\left\{u_{n}\right\}$ is bounded in $H^{1}\left(\mathbb{R}^{3}\right)$, which implies that there exist $u_{b} \in H^{1}\left(\mathbb{R}^{3}\right)$ and a subsequence, still denoted by $\left\{u_{n}\right\}$, such that $u_{n} \rightarrow u_{b}$ as well as $u_{n}^{ \pm} \rightarrow$ $u_{b}^{ \pm}$weakly in $H^{1}\left(\mathbb{R}^{3}\right)$.

By $\left\{u_{n}\right\} \subset M_{b}$, it follows from $\left\langle J_{b}^{\prime}\left(u_{n}\right), u_{n}^{ \pm}\right\rangle=0$ that

$$
\begin{aligned}
\left\|u_{n}^{ \pm}\right\|^{2} & \leq\left\|u_{n}^{ \pm}\right\|^{2}+b\left|\nabla u_{n}\right|_{2}^{2}\left|\nabla u_{n}^{ \pm}\right|_{2}^{2}+\int_{\mathbb{R}^{3}} k(x) \phi_{u_{n}}\left(u_{n}^{ \pm}\right)^{2} d x+\lambda\left|u_{n}^{ \pm}\right|_{p}^{p} \\
& =\int_{\mathbb{R}^{3}} h(x)\left|u_{n}^{ \pm}\right|^{q} d x \leq|h|_{\frac{6}{6-q_{1}}} S_{r}^{-q}\left\|u_{n}^{ \pm}\right\|^{q}
\end{aligned}
$$

where $r=\frac{6 q}{q_{1}}$. Therefore,

$$
\left\|u_{n}^{ \pm}\right\| \geq c_{0}>0
$$

Thus, by (2.25)-(2.26) and Lemma 2.3, we obtain $0<c_{0}^{2} \leq \lim _{n \rightarrow \infty} \int_{\mathbb{R}^{3}} h(x)\left|u_{n}^{ \pm}\right|^{q} d x=$ $\int_{\mathbb{R}^{3}} h(x)\left|u_{b}^{ \pm}\right|^{q} d x$, which implies that $u_{b}^{ \pm} \neq 0$ taking into account the assumption $h(x)>0$, a.e. $x \in \mathbb{R}^{3}$.

Again, by the weak semi-continuity of the norm and Lemma 2.3, we get

$$
\begin{aligned}
& \left\|u_{b}^{ \pm}\right\|^{2}+b\left|\nabla u_{b}\right|_{2}^{2}\left|\nabla u_{b}^{ \pm}\right|_{2}^{2}+\int_{\mathbb{R}^{3}} k(x) \phi_{u_{b}}\left(u_{b}^{ \pm}\right)^{2} d x+\lambda\left|u_{b}^{ \pm}\right|_{p}^{p} \\
& \quad \leq \lim _{n \rightarrow \infty} \inf \left\{|| u_{n}^{ \pm} \|^{2}+b\left|\nabla u_{n}\right|_{2}^{2}\left|\nabla u_{n}^{ \pm}\right|_{2}^{2}+\int_{\mathbb{R}^{3}} k(x) \phi_{u_{n}}\left(u_{n}^{ \pm}\right)^{2} d x+\lambda\left|u_{n}^{ \pm}\right|_{p}^{p}\right\} \\
& \quad=\lim _{n \rightarrow \infty} \inf \left\{\int_{\mathbb{R}^{3}} h(x)\left|u_{n}^{ \pm}\right|^{q} d x\right\}=\int_{\mathbb{R}^{3}} h(x)\left|u_{b}^{ \pm}\right|^{q} d x,
\end{aligned}
$$

which means that $\varphi_{1}(1,1) \leq 0$ and $\psi_{1}(1,1) \leq 0$ in (2.13) corresponding to $u_{b}$. Hence, by Lemma 2.5 , there exists an unique $(s, t) \in(0,1] \times(0,1]$ with $u_{b}^{*}=s u_{b}^{+}+t u_{b}^{-} \in M_{b}$. Moreover, by the weak semi-continuity of the norm and Lemmas $2.2-2.3$ and (2.7), we have

$$
m_{b} \leq J_{b}\left(u_{b}^{*}\right)-\frac{1}{q}\left\langle J_{b}^{\prime}\left(u_{b}^{*}\right), u_{b}^{*}\right\rangle
$$


Chai and Liu Boundary Value Problems

(2019) 2019:160

Page 14 of 25

$$
\begin{aligned}
& =\left(\frac{1}{2}-\frac{1}{q}\right)\left\|u_{b}^{*}\right\|^{2}+\left(\frac{1}{4}-\frac{1}{q}\right) b\left|\nabla u_{b}^{*}\right|_{2}^{4}+\left(\frac{1}{4}-\frac{1}{q}\right) \int_{\mathbb{R}^{3}} k(x) \phi_{u_{b}^{*}}\left(u_{b}^{*}\right)^{2} d x \\
& +\lambda\left(\frac{1}{p}-\frac{1}{q}\right)\left|u_{b}^{*}\right|_{p}^{p} \\
& =\left(\frac{1}{2}-\frac{1}{q}\right)\left[s^{2}\left\|u_{b}^{+}\right\|^{2}+t^{2}\left\|u_{b}^{-}\right\|^{2}\right] \\
& +\left(\frac{1}{4}-\frac{1}{q}\right) b\left[s^{4}\left|\nabla u_{b}^{+}\right|_{2}^{4}+t^{4}\left|\nabla u_{b}^{-}\right|_{2}^{4}+2 s^{2} t^{2}\left|\nabla u_{b}^{+}\right|_{2}^{4}\left|\nabla u_{b}^{-}\right|_{2}^{4}\right] \\
& +\left(\frac{1}{4}-\frac{1}{q}\right)\left[s^{4} \int_{\mathbb{R}^{3}} k(x) \phi_{u_{b}^{+}}\left(u_{b}^{+}\right)^{2} d x+t^{4} \int_{\mathbb{R}^{3}} k(x) \phi_{u_{b}^{-}}\left(u_{b}^{-}\right)^{2} d x\right. \\
& \left.+2 s^{2} t^{2} \int_{\mathbb{R}^{3}} k(x) \phi_{u_{b}^{+}}\left(u_{b}^{-}\right)^{2} d x\right] \\
& +\lambda\left(\frac{1}{p}-\frac{1}{q}\right)\left(s^{p}\left|u_{b}^{+}\right|_{p}^{p}+t^{p}\left|u_{b}^{-}\right|_{p}^{p}\right) \\
& \leq\left(\frac{1}{2}-\frac{1}{q}\right)\left(\left\|u_{b}^{+}\right\|^{2}+\left\|u_{b}^{-}\right\|^{2}\right)+\left(\frac{1}{4}-\frac{1}{q}\right) b\left[\left|\nabla u_{b}^{+}\right|_{2}^{4}+\left|\nabla u_{b}^{-}\right|_{2}^{4}+2\left|\nabla u_{b}^{+}\right|_{2}^{2}\left|\nabla u_{b}^{-}\right|_{2}^{2}\right] \\
& +\left(\frac{1}{4}-\frac{1}{q}\right)\left[\int_{\mathbb{R}^{3}} k(x) \phi_{u_{b}^{+}}\left(u_{b}^{+}\right)^{2} d x+\int_{\mathbb{R}^{3}} k(x) \phi_{u_{b}^{-}}\left(u_{b}^{-}\right)^{2} d x+2 \int_{\mathbb{R}^{3}} k(x) \phi_{u_{b}^{+}}\left(u_{b}^{-}\right)^{2} d x\right] \\
& +\lambda\left(\frac{1}{p}-\frac{1}{q}\right)\left(\left|u_{b}^{+}\right|_{p}^{p}+\left|u_{b}^{-}\right|_{p}^{p}\right) \\
& \leq \lim _{n \rightarrow \infty} \inf \left\{\left(\frac{1}{2}-\frac{1}{q}\right)\left(\left\|u_{n}^{+}\right\|^{2}+\left\|u_{n}^{-}\right\|^{2}\right)\right. \\
& +\left(\frac{1}{4}-\frac{1}{q}\right) b\left[\left|\nabla u_{n}^{+}\right|_{2}^{4}+\left|\nabla u_{n}^{-}\right|_{2}^{4}+2\left|\nabla u_{n}^{+}\right|_{2}^{2}\left|\nabla u_{n}^{-}\right|_{2}^{2}\right] \\
& +\left(\frac{1}{4}-\frac{1}{q}\right)\left[\int_{\mathbb{R}^{3}} k(x) \phi_{u_{n}^{+}}\left(u_{n}^{+}\right)^{2} d x+\int_{\mathbb{R}^{3}} k(x) \phi_{u_{n}^{-}}\left(u_{n}^{-}\right)^{2} d x+2 \int_{\mathbb{R}^{3}} k(x) \phi_{u_{n}^{+}}\left(u_{n}^{-}\right)^{2} d x\right] \\
& \left.+\lambda\left(\frac{1}{p}-\frac{1}{q}\right)\left(\left|u_{n}^{+}\right|_{p}^{p}+\left|u_{n}^{-}\right|_{p}^{p}\right)\right\} \\
& =\lim _{n \rightarrow \infty} \inf \left[J_{b}\left(u_{n}\right)-\frac{1}{q}\left\langle J_{b}^{\prime}\left(u_{n}\right), u_{n}\right\rangle\right]=\lim _{n \rightarrow \infty} \inf \left[J_{b}\left(u_{n}\right)\right]=m_{b} \text {. }
\end{aligned}
$$

Thus, $s=t=1$, and therefore, $u_{b} \in M_{b}, J_{b}\left(u_{b}\right)=m_{b}$.

Lemma 2.7 For any given $u \in H^{1}\left(\mathbb{R}^{3}\right)$ with $u^{ \pm} \neq 0$. Let $g(s, t)=J_{b}\left(s u^{+}+t u^{-}\right),(s, t) \in$ $\mathbb{R}_{+} \times \mathbb{R}_{+}$, then there exists an unique pair $\left(s_{u}, t_{u}\right) \in \mathbb{R}_{+}^{0} \times \mathbb{R}_{+}^{0}$ such that $g$ attains its global maximum on $\mathbb{R}_{+} \times \mathbb{R}_{+}$at the point $\left(s_{u}, t_{u}\right)$, which is exactly obtained in Lemma 2.4.

Proof Because $2 \leq p \leq 4<q<6, \lambda \geq 0$ and $h(x)>0$, are. $x \in \mathbb{R}^{3}$, we can know that $g(s, t) \rightarrow$ $-\infty$ as $|(s, t)| \rightarrow \infty$. Hence, we only need to prove that $g$ cannot achieve its maximum on the boundary of $\mathbb{R}_{+} \times \mathbb{R}_{+}$. In fact, if not-if $g$ achieved its maximum at point $(0, \bar{t}), \bar{t}>0$, then, by

$$
\begin{aligned}
g(s, \bar{t}) & =J_{b}\left(s u^{+}+\bar{t} u^{-}\right) \\
& =\frac{s^{2}}{2}\left\|u^{+}\right\|^{2}+\frac{s^{4}}{4} b\left|\nabla u^{+}\right|_{2}^{4}+\frac{s^{4}}{4} \int_{\mathbb{R}^{3}} k(x) \phi_{u^{+}}\left(u^{+}\right)^{2} d x+\frac{\lambda}{p} s^{p}\left|u^{+}\right|_{p}^{p}
\end{aligned}
$$




$$
\begin{aligned}
& -\frac{s^{q}}{q} \int_{\mathbb{R}^{3}} h(x)\left|u^{+}\right|^{q} d x \\
& +\frac{\bar{t}^{2}}{2}\left\|u^{-}\right\|^{2}+\frac{\bar{t}^{4}}{4} b\left|\nabla u^{-}\right|_{2}^{4}+\frac{\bar{t}}{4} \int_{\mathbb{R}^{3}} k(x) \phi_{u^{-}}\left(u^{-}\right)^{2} d x+\frac{\lambda}{p} \bar{t}^{p}\left|u^{-}\right|_{p}^{p} \\
& -\frac{\bar{t}^{q}}{q} \int_{\mathbb{R}^{3}} h(x)\left|u^{-}\right|^{q} d x \\
& +2 s^{2} \bar{t}^{2}\left(b\left|\nabla u^{+}\right|_{2}^{4}\left|\nabla u^{-}\right|_{2}^{4}+\int_{\mathbb{R}^{3}} k(x) \phi_{u^{+}}\left(u^{-}\right)^{2} d x\right) \geq g(s, 0)+g(0, \bar{t}),
\end{aligned}
$$

and the fact that $g(s, 0)>0$ as $s>0$ is small enough, we know that $g(s, \bar{t})>g(0, \bar{t})$ as $s>0$ is small enough, which contradicts the assumption that $g$ achieved its maximum value at $(0, \bar{t})$ on $\mathbb{R}_{+} \times \mathbb{R}_{+}$. So, $g$ achieved its maximum in some point $\left(s_{u}, t_{u}\right) \in \mathbb{R}_{+}^{0} \times \mathbb{R}_{+}^{0}$ on $\mathbb{R}_{+} \times \mathbb{R}_{+}$. Of course, $\left(s_{u}, t_{u}\right)$ is a critical point of $g$ on $\mathbb{R}_{+} \times \mathbb{R}_{+}$, and therefore it follows from the proof of Lemma 2.4 that the pair $\left(s_{u}, t_{u}\right)$ is the unique solution of systems $(2.11)$ in $\mathbb{R}_{+}^{0} \times \mathbb{R}_{+}^{0}$.

\section{Main results}

We are now in a position to give our main results in this paper.

Theorem 3.1 Assume that conditions $(l),(k),(h)$ hold, then problem (1.1) possesses one least energy sign-changing solution $u_{b}$, which has exactly two nodal domains, where $u_{b}$ is given in Lemma 2.6.

Proof We apply the quantitative deformation lemma to prove that $J_{b}^{\prime}\left(u_{b}\right)=0$.

Owing to the fact that $u_{b} \in M_{b}$ and $J_{b}\left(u_{b}\right)=m_{b}$, in terms of Lemma 2.7, for any $(s, t) \in$ $\mathbb{R}_{+}^{0} \times \mathbb{R}_{+}^{0}$ with $(s, t) \neq(1,1)$, we immediately obtain

$$
J_{b}\left(s u_{b}^{+}+t u_{b}^{-}\right)<J_{b}\left(u_{b}^{+}+u_{b}^{-}\right)=m_{b}
$$

If $J_{b}^{\prime}\left(u_{b}\right) \neq 0$, then there exist $r>0$ and $\tau>0$ satisfying

$$
\left\|J_{b}^{\prime}(u)\right\|>\tau \quad \text { as }\left\|u-u_{b}\right\|<3 r
$$

Set $U=\left(1-\sigma_{0}, 1+\sigma_{0}\right) \times\left(1-\sigma_{0}, 1+\sigma_{0}\right)$, where $0<\sigma_{0}<\frac{1}{2}$, and $h(s, t)=s u_{b}^{+}+t u_{b}^{-},(s, t) \in \bar{U}$. It follows from (3.1) that

$$
m^{*}:=\max _{\partial U} J_{b} \circ h=\max _{\partial U} J_{b}\left(s u_{b}^{+}+t u_{b}^{-}\right)<m_{b}
$$

Take $0<\varepsilon<\min \left\{\left(m_{b}-m^{*}\right) / 2, \tau r / 8\right\}, S=\left\{u \in H^{1}\left(\mathbb{R}^{3}\right):\left\|u-u_{b}\right\|<r\right\}$.

Let $S_{2 r}=\left\{u \in H^{1}\left(\mathbb{R}^{3}\right): \operatorname{dist}(u, S) \leq 2 r\right\}$. Applying Lemma 2.3 in [33], there exists a deformation $\eta$ satisfying that

(i) $\eta(1, u)=u$, if $u \notin J_{b}^{-1}\left(m_{b}-2 \varepsilon, m_{b}+2 \varepsilon\right) \cap S_{2 r}$;

(ii) $\eta\left(1, J_{b}^{m_{b}+\varepsilon} \cap S\right) \subset J_{b}^{m_{b}-\varepsilon}$;

(iii) $J_{b}(\eta(1, u)) \leq J_{b}(u)$ for any $u \in H^{1}\left(\mathbb{R}^{3}\right)$.

Now, we show that

$$
\eta(1, h(U)) \cap M_{b} \neq \varnothing
$$

which will contradict the definition of $m_{b}$. 
First, we claim that

$$
\max _{(s, t) \in \bar{U}} J_{b}(\eta(1, h(s, t)))<m_{b}
$$

In fact, for any $(s, t) \in \bar{U}$, we consider the following two cases:

(a) If $(s, t) \neq(1,1)$, then, by (3.3) together with (iii) above, we have

$$
J_{b}(\eta(1, h(s, t))) \leq J_{b}(h(s, t))=J_{b}\left(s u_{b}^{+}+t u_{b}^{-}\right)<m_{b} .
$$

(b) If $(s, t)=(1,1)$, then, by $J_{b}(h(1,1))=J_{b}\left(u_{b}\right)=m_{b}<m_{b}+\varepsilon$ and $h(1,1)=u_{b} \in S$, it follows from (ii) that

$$
J_{b}(\eta(1, h(1,1))) \leq m_{b}-\varepsilon<m_{b}
$$

Hence, from the above arguments (a) and (b), we know that (3.3) is true.

Secondly, for $(s, t) \in \bar{U}$, let $\varphi(s, t)=\eta(1, h(s, t))$ and

$$
\phi(s, t)=\left(\phi_{1}(s, t), \phi_{2}(s, t)\right), \quad \psi(s, t)=\left(\psi_{1}(s, t), \psi_{2}(s, t)\right),
$$

where

$$
\begin{aligned}
& \phi_{1}(s, t)=\left\langle J_{b}^{\prime}(h(s, t)), u_{b}^{+}\right\rangle, \quad \phi_{2}(s, t)=\left\langle J_{b}^{\prime}(h(s, t)), u_{b}^{-}\right\rangle \\
& \psi_{1}(s, t)=\frac{1}{s}\left\langle J_{b}^{\prime}(\varphi(s, t)), \varphi^{+}(s, t)\right\rangle, \quad \psi_{2}(s, t)=\frac{1}{t}\left\langle J_{b}^{\prime}(\varphi(s, t)), \varphi^{-}(s, t)\right\rangle .
\end{aligned}
$$

Since $u_{b} \in M_{b}$, by Lemma $2.4, \phi(s, t)=0 \Leftrightarrow(s, t)=(1,1)$. Again, by the proof of Lemma 2.4, we know that $\left.\frac{\partial\left(\phi_{1}, \phi_{2}\right)}{\partial(s, t)}\right|_{(1,1)}>0$, and therefore, the degree theory yields

$$
\operatorname{deg}(\phi, U, \theta)=1
$$

where $\theta=(0,0)$. On the other hand, for any $(s, t) \in \partial U$, by (3.2) and $0<\varepsilon<\frac{m_{b}-m^{*}}{2}$, we have

$$
J_{b}(h(s, t)) \leq m^{*}<m_{b}-2 \varepsilon
$$

Thus, $h(s, t) \notin J_{b}^{-1}\left(m_{b}-2 \varepsilon, m_{b}+2 \varepsilon\right) \cap S_{2 r}$. So, the conclusion (i) above implies that

$$
\varphi(s, t)=\eta(1, h(s, t))=h(s, t), \quad \text { for any }(s, t) \in \partial U
$$

which means that $\phi(s, t)=\psi(s, t)$, for any $(s, t) \in \partial U$. Hence, $\operatorname{deg}(\psi, U, \theta)=\operatorname{deg}(\phi, U, \theta)=1$. Therefore, there exists $\left(s_{0}, t_{0}\right) \in U$ such that $\psi\left(s_{0}, t_{0}\right)=0$. That means that $\eta\left(1, h\left(s_{0}, t_{0}\right)\right)=$ $\varphi\left(s_{0}, t_{0}\right) \in M_{b}$. Hence, $J_{b}\left(1, h\left(s_{0}, t_{0}\right)\right) \geq m_{b}$, which contradicts (3.3). Thus, we have deduced that $J_{b}^{\prime}\left(u_{b}\right)=0, u_{b} \in M_{b}$, and $u_{b}$ is a least energy sign-changing solution of problem (1.1).

Finally, we show that $u_{b}$ has exactly two nodal domains. To this end, let $u_{b}=u_{1}+u_{2}+u_{3}$, satisfying that $u_{1}(x) \geq 0, u_{2}(x) \leq 0$, for any $x \in \mathbb{R}^{3} ; u_{1}(x)=u_{2}(x)=0$, for any $x \in \mathbb{R}^{3} \backslash\left(\Omega_{1} \cup\right.$ $\left.\Omega_{2}\right) ; u_{3}(x)=0$, for any $x \in \Omega_{1} \cup \Omega_{2}$, where $\Omega_{1}=\left\{x \in \mathbb{R}^{3} \mid u_{1}(x)>0\right\}, \Omega_{2}=\left\{x \in \mathbb{R}^{3} \mid u_{2}(x)<0\right\}$ are connected open subsets of $\Omega$. 
Set $u=u_{1}+u_{2}$. Then $u^{+}=u_{1}$ and $u^{-}=u_{2}$ with $u^{ \pm} \neq 0$.

In the following, we deduce that $u_{3}=0$.

Suppose by contradiction that $u_{3} \neq 0$. Then, by $J_{b}^{\prime}\left(u_{b}\right) u_{1}=0$, we can deduce that $\varphi_{1}(1,1) \leq 0$. Similarly, by $J_{b}^{\prime}\left(u_{b}\right) u_{2}=0$, we can deduce that $\psi_{1}(1,1) \leq 0$, where $\varphi_{1}, \psi_{1}$ are given by (2.13) with $u^{+}=u_{1}, u^{-}=u_{2}$. So, by Lemmas 2.4-2.5, there exists an unique pair $\left(s_{u}, t_{u}\right) \in(0,1] \times(0,1]$ such that $s_{u} u^{+}+t_{u} u^{-} \in M_{b}$. Then

$$
m_{b} \leq J_{b}\left(s_{u} u_{1}+t_{u} u_{2}\right), \quad\left\langle J_{b}^{\prime}\left(s_{u} u_{1}+t_{u} u_{2}\right), s_{u} u_{1}+t_{u} u_{2}\right\rangle=0 .
$$

Consequently,

$$
\begin{aligned}
m_{b} \leq & J_{b}\left(s_{u} u_{1}+t_{u} u_{2}\right)=J_{b}\left(s_{u} u_{1}+t_{u} u_{2}\right)-\frac{1}{q}\left\langle J_{b}^{\prime}\left(s_{u} u_{1}+t_{u} u_{2}\right), s_{u} u_{1}+t_{u} u_{2}\right\rangle \\
= & \left(\frac{1}{2}-\frac{1}{q}\right)\left(s_{u}^{2}\left\|u_{1}\right\|^{2}+t_{u}^{2}\left\|u_{2}\right\|^{2}\right)+\left(\frac{1}{4}-\frac{1}{q}\right) b\left[s_{u}^{2}\left|\nabla u_{1}\right|_{2}^{2}+t_{u}^{2}\left|\nabla u_{2}\right|_{2}^{2}\right]^{2} \\
& +\left(\frac{1}{4}-\frac{1}{q}\right) \int_{\mathbb{R}^{3}} k(x)\left[s_{u}^{4} \phi_{u_{1}}\left(u_{1}\right)^{2}+t_{u}^{4} \phi_{u_{2}}\left(u_{2}\right)^{2}+2 s_{u}^{2} t_{u}^{2} \phi_{u_{1}}\left(u_{2}\right)^{2}\right] d x \\
& +\lambda\left(\frac{1}{p}-\frac{1}{q}\right)\left(s_{u}^{p}\left|u_{1}\right|_{p}^{p}+t_{u}^{p}\left|u_{2}\right|_{p}^{p}\right) \\
\leq & \left(\frac{1}{2}-\frac{1}{q}\right)\left(\left\|u_{1}\right\|^{2}+|| u_{2} \|^{2}\right)+\left(\frac{1}{4}-\frac{1}{q}\right) b\left[\left|\nabla u_{1}\right|_{2}^{2}+\left|\nabla u_{2}\right|_{2}^{2}\right]^{2} \\
& +\left(\frac{1}{4}-\frac{1}{q}\right) \int_{\mathbb{R}^{3}} k(x)\left[\phi_{u_{1}}\left(u_{1}\right)^{2}+\phi_{u_{2}}\left(u_{2}\right)^{2}+2 \phi_{u_{1}}\left(u_{2}\right)^{2}\right] d x \\
& +\lambda\left(\frac{1}{p}-\frac{1}{q}\right)\left(\left|u_{1}\right|_{p}^{p}+\left|u_{2}\right|_{p}^{p}\right) \\
= & J_{b}\left(u_{1}+u_{2}\right)-\frac{1}{q}\left\langle J_{b}^{\prime}\left(u_{1}+u_{2}\right), u_{1}+u_{2}\right\rangle \\
= & J_{b}\left(u_{b}\right)-\frac{1}{q}\left\langle J_{b}^{\prime}\left(u_{b}\right), u_{1}+u_{2}\right\rangle \\
& -\left[J_{b}\left(u_{3}\right)+\left(\frac{1}{2}-\frac{1}{q}\right)\left(b\left|\nabla\left(u_{1}+u_{2}\right)\right|_{2}^{2}\left|\nabla u_{3}\right|_{2}^{2}+\int_{\mathbb{R}^{3}} k(x) \phi_{u_{1}+u_{2}}\left(u_{3}\right)^{2} d x\right)\right] \\
= & J_{b}\left(u_{b}\right)-\left[J_{b}\left(u_{3}\right)+\left(\frac{1}{2}-\frac{1}{q}\right)\left(b\left|\nabla\left(u_{1}+u_{2}\right)\right|_{2}^{2}\left|\nabla u_{3}\right|_{2}^{2}\right.\right. \\
& \left.\left.+\int_{\mathbb{R}^{3}} k(x) \phi_{u_{1}+u_{2}}\left(u_{3}\right)^{2} d x\right)\right] . \\
&
\end{aligned}
$$

On the other hand, by the fact that $u_{3} \neq 0,2 \leq p \leq 4<q<6, \lambda \geq 0$, we have

$$
\begin{aligned}
J_{b}\left(u_{3}\right)= & J_{b}\left(u_{3}\right)-\frac{1}{q}\left\langle J_{b}^{\prime}\left(u_{b}\right), u_{3}\right\rangle \\
= & \left(\frac{1}{2}-\frac{1}{q}\right)\left\|u_{3}\right\|^{2}+\left(\frac{1}{4}-\frac{1}{q}\right) b\left|\nabla u_{3}\right|_{2}^{4}+\left(\frac{1}{4}-\frac{1}{q}\right) \int_{\mathbb{R}^{3}} k(x) \phi_{u_{3}}\left(u_{3}\right)^{2} d x \\
& +\lambda\left(\frac{1}{p}-\frac{1}{q}\right)\left|u_{3}\right|_{p}^{p}-\frac{b}{q}\left|\nabla\left(u_{1}+u_{2}\right)\right|_{2}^{2}\left|\nabla u_{3}\right|_{2}^{2}-\frac{1}{q} \int_{\mathbb{R}^{3}} k(x) \phi_{u_{1}+u_{2}}\left(u_{3}\right)^{2} d x \\
> & -\frac{b}{q}\left|\nabla\left(u_{1}+u_{2}\right)\right|_{2}^{2}\left|\nabla u_{3}\right|_{2}^{2}-\frac{1}{q} \int_{\mathbb{R}^{3}} k(x) \phi_{u_{1}+u_{2}}\left(u_{3}\right)^{2} d x .
\end{aligned}
$$


Thus,

$$
J_{b}\left(u_{3}\right)+\frac{1}{q}\left(b\left|\nabla\left(u_{1}+u_{2}\right)\right|_{2}^{2}\left|\nabla u_{3}\right|_{2}^{2}+\int_{\mathbb{R}^{3}} k(x) \phi_{u_{1}+u_{2}}\left(u_{3}\right)^{2} d x\right)>0 .
$$

The condition $q>4$ shows that $\frac{1}{2}-\frac{1}{q}>\frac{1}{q}$, and therefore, it follows from the above inequality that

$$
J_{b}\left(u_{3}\right)+\left(\frac{1}{2}-\frac{1}{q}\right)\left(b\left|\nabla\left(u_{1}+u_{2}\right)\right|_{2}^{2}\left|\nabla u_{3}\right|_{2}^{2}+\int_{\mathbb{R}^{3}} k(x) \phi_{u_{1}+u_{2}}\left(u_{3}\right)^{2} d x\right)>0
$$

By (3.4)-(3.5), we have

$$
m_{b}<J_{b}\left(u_{b}\right)=m_{b}
$$

which is a contradiction. Hence, $u_{3}=0$, and therefore, $u_{b}$ has exactly two nodal domains.

In the following, we always assume that $b>0$ in problem (1.1). We will investigate the convergence of $u_{b}$ as $b \searrow 0$.

Theorem 3.2 Assume that the conditions $(l),(k),(h)$ hold. Then, for any sequence $\left\{b_{n}\right\}$ with $b_{n} \searrow 0$ as $n \rightarrow \infty$, there exists a subsequence, still denoted by $\left\{b_{n}\right\}$ such that $u_{b_{n}} \rightarrow$ $u_{0} \in H^{1}\left(\mathbb{R}^{3}\right)$ in $H^{1}\left(\mathbb{R}^{3}\right)$ as $n \rightarrow \infty$. Moreover, $u_{0}$ is a least energy sign-changing solution of problem (1.2).

Proof For any sequence $\left\{b_{n}\right\}$ with $b_{n} \searrow 0$ as $n \rightarrow \infty, u_{b_{n}}$ is one least energy sign-changing solution of problem (1.1) corresponding to $b=b_{n}$.

(1) Firstly, we show that $\left\{u_{b_{n}}\right\}$ is bounded in $H^{1}\left(\mathbb{R}^{3}\right)$.

Take a nonzero function $g \in C_{0}^{\infty}\left(\mathbb{R}^{3}\right)$ with $g^{ \pm} \neq 0$. Because $2 \leq p \leq 4<q<6, \lambda \geq 0$, and $h(x)>0$, a.e. $x \in \mathbb{R}^{3}$, we can choose an appropriate positive number $\tau>0$ such that

$$
\varphi(1,1) \leq 0, \quad \psi(1,1) \leq 0
$$

holds for all $b \in[0,1]$ corresponding to $u^{+}=\tau g^{+}, u^{-}=\tau g^{-}$in (2.13). Thus, by Lemma 2.5, for each $b \in(0,1]$, there exists a pair $\left(s_{b}, t_{b}\right) \in(0,1] \times(0,1]$ such that $s_{b} u^{+}+t_{b} u^{-} \in M_{b}$. Let $\bar{u}:=s_{b} u^{+}+t_{b} u^{-}$and $u_{b}$ be a least energy sign-changing solution of problem (1.1), then

$$
\begin{aligned}
J_{b}\left(u_{b}\right) \leq & J_{b}(\bar{u})=J_{b}(\bar{u})-\frac{1}{q}\left\langle J_{b}^{\prime}(\bar{u}), \bar{u}\right\rangle \\
= & \left(\frac{1}{2}-\frac{1}{q}\right)\left(s_{b}\left\|u^{+}\right\|^{2}+t_{b}\left\|u^{-}\right\|^{2}\right)+\left(\frac{1}{4}-\frac{1}{q}\right) b\left[s_{b}^{2}\left|\nabla u^{+}\right|_{2}^{2}+t_{b}^{2}\left|\nabla u^{-}\right|_{2}^{2}\right]^{2} \\
& +\left(\frac{1}{4}-\frac{1}{q}\right) \int_{\mathbb{R}^{3}} k(x)\left[s_{b}^{4} \phi_{u^{+}}\left(u^{+}\right)^{2}+t_{b}^{4} \phi_{u^{-}}\left(u^{-}\right)^{2}+2 s_{b}^{2} t_{b}^{2} \phi_{u^{+}}\left(u^{-}\right)^{2}\right] d x \\
& +\lambda\left(\frac{1}{p}-\frac{1}{q}\right)\left(s_{b}^{p}\left|u^{+}\right|_{p}^{p}+t_{b}^{p}\left|u^{-}\right|_{p}^{p}\right) \\
\leq & \left(\frac{1}{2}-\frac{1}{q}\right)\left(\left\|u^{+}\right\|^{2}+\left\|u^{-}\right\|^{2}\right)+\left(\frac{1}{4}-\frac{1}{q}\right)\left[\left|\nabla u^{+}\right|_{2}^{2}+\left|\nabla u^{-}\right|_{2}^{2}\right]^{2}
\end{aligned}
$$




$$
\begin{aligned}
& +\lambda\left(\frac{1}{p}-\frac{1}{q}\right)\left(\left|u^{+}\right|_{p}^{p}+\left|u^{-}\right|_{p}^{p}\right) \\
& +\left(\frac{1}{4}-\frac{1}{q}\right)\left[\int_{\mathbb{R}^{3}} k(x)\left(\phi_{u^{+}}\left(u^{+}\right)^{2}+\phi_{u^{-}}\left(u^{-}\right)^{2}+2 \phi_{u^{+}}\left(u^{-}\right)^{2}\right) d x\right]:=M_{0} .
\end{aligned}
$$

Thus, $J_{b}\left(u_{b}\right) \leq M_{0}$ for all $b \in[0,1]$.

Owing to the fact that $b_{n} \searrow 0$, we can assume $b_{n} \in(0,1]$, and therefore, $J_{b_{n}}\left(u_{b_{n}}\right) \leq M_{0}$ for all $n \geq 1$. On the other hand, for all $n \geq 1$,

$$
M_{0} \geq J_{b_{n}}\left(u_{b_{n}}\right)=J_{b_{n}}\left(u_{b_{n}}\right)-\frac{1}{q}\left\langle J_{b_{n}}^{\prime}\left(u_{b_{n}}\right), u_{b_{n}}\right\rangle \geq\left(\frac{1}{2}-\frac{1}{q}\right)\left\|u_{b_{n}}\right\|^{2} .
$$

Hence, $\left\{u_{b_{n}}\right\}$ is bounded in $H^{1}\left(\mathbb{R}^{3}\right)$.

(2) Because $\left\{u_{b_{n}}\right\}$ is bounded in $H^{1}\left(\mathbb{R}^{3}\right)$, there exists a subsequence of $\left\{b_{n}\right\}$, still denoted by $\left\{b_{n}\right\}$, such that $u_{b_{n}} \rightarrow u_{0}$ as well as $u_{b_{n}}^{ \pm} \rightarrow u_{0}^{ \pm}$weakly in $H^{1}\left(\mathbb{R}^{3}\right)$. Then $u_{b_{n}} \rightarrow u_{0}$ in $L_{\text {loc }}^{r}\left(\mathbb{R}^{3}\right)$ for $r \in[1,6)$ and $u_{b_{n}}(x) \rightarrow u_{0}(x)$ a.e. $x \in \mathbb{R}^{3}$.

Now, we show that $u_{b_{n}} \rightarrow u_{0}$ in $H^{1}\left(\mathbb{R}^{3}\right)$.

In fact, from $u_{b_{n}} \rightarrow u$ weakly in $H^{1}\left(\mathbb{R}^{3}\right)$ and $J_{b_{n}}^{\prime}\left(u_{b_{n}}\right)=0$, it follows that

$$
\left\langle J_{b_{n}}^{\prime}\left(u_{b_{n}}\right)-J_{0}^{\prime}\left(u_{0}\right), u_{b_{n}}-u_{0}\right\rangle=-\left\langle J_{0}^{\prime}\left(u_{0}\right), u_{b_{n}}-u_{0}\right\rangle \rightarrow 0 \quad \text { as } n \rightarrow \infty
$$

On the other hand, we have

$$
\begin{aligned}
\left\|u_{b_{n}}-u_{0}\right\|^{2}= & \left\langle J_{b_{n}}^{\prime}\left(u_{b_{n}}\right)-J_{0}^{\prime}\left(u_{0}\right), u_{b_{n}}-u_{0}\right\rangle-d_{n} \\
& -\int_{\mathbb{R}^{3}} k(x) \phi_{u_{b_{n}}}\left(u_{b_{n}}-u_{0}\right)^{2} d x-\lambda \int_{\mathbb{R}^{3}}\left|u_{b_{n}}\right|^{p-2}\left(u_{b_{n}}-u_{0}\right)^{2} d x \\
\leq & \left\langle J_{b_{n}}^{\prime}\left(u_{b_{n}}\right)-J_{0}^{\prime}\left(u_{0}\right), u_{b_{n}}-u_{0}\right\rangle-d_{n},
\end{aligned}
$$

where

$$
\begin{aligned}
d_{n}= & b_{n}\left|\nabla u_{b_{n}}\right|_{2}^{2} \int_{\mathbb{R}^{3}} \nabla u_{b_{n}} \cdot\left(\nabla u_{b_{n}}-\nabla u_{0}\right) d x+\int_{\mathbb{R}^{3}} k(x)\left(\phi_{u_{b_{n}}}-\phi_{u_{0}}\right) u_{0}\left(u_{b_{n}}-u_{0}\right) d x \\
& +\lambda \int_{\mathbb{R}^{3}}\left(\left|u_{b_{n}}\right|^{p-2}-\left|u_{0}\right|^{p-2}\right) u_{0}\left(u_{b_{n}}-u_{0}\right) d x \\
& -\int_{\mathbb{R}^{3}} h(x)\left|u_{b_{n}}\right|^{q-2} u_{b_{n}}\left(u_{b_{n}}-u_{0}\right) d x+\int_{\mathbb{R}^{3}} h(x)\left|u_{0}\right|^{q-2} u_{0}\left(u_{b_{n}}-u_{0}\right) d x .
\end{aligned}
$$

In the following, we deduce that $d_{n} \rightarrow 0$ as $n \rightarrow \infty$.

Owing to the fact that $\left\{u_{b_{n}}\right\}$ is bounded in $H^{1}\left(\mathbb{R}^{3}\right)$, it is easy to verify that the sequence $\left\{\left|\nabla u_{b_{n}}\right|_{2}^{2} \int_{\mathbb{R}^{3}} \nabla u_{b_{n}} \cdot\left(\nabla u_{b_{n}}-\nabla u_{0}\right) d x\right\}$ is bounded, and therefore

$$
b_{n}\left|\nabla u_{b_{n}}\right|_{2}^{2} \int_{\mathbb{R}^{3}} \nabla u_{b_{n}} \cdot\left(\nabla u_{b_{n}}-\nabla u_{0}\right) d x \rightarrow 0
$$

as $n \rightarrow \infty$ noting that $b_{n} \rightarrow 0$ as $n \rightarrow \infty$.

Now, we prove that

$$
\int_{\mathbb{R}^{3}}\left(\left|u_{b_{n}}\right|^{p-2}-\left|u_{0}\right|^{p-2}\right) u_{0}\left(u_{b_{n}}-u_{0}\right) d x \rightarrow 0 \quad \text { as } n \rightarrow \infty .
$$


Chai and Liu Boundary Value Problems

(2019) 2019:160

Page 20 of 25

For $R>0$, let $\Omega_{R}=\left\{x \in \mathbb{R}^{3}:\|x\|<R\right\}, \Omega_{R}^{C}=\left\{x \in \mathbb{R}^{3}:\|x\| \geq R\right\}$, we have

$$
\begin{aligned}
A_{n} & :=\left|\int_{\Omega_{R}^{C}}\left(\left|u_{b_{n}}\right|^{p-2}-\left|u_{0}\right|^{p-2}\right) u_{0}\left(u_{b_{n}}-u_{0}\right) d x\right| \\
& \leq \int_{\Omega_{R}^{C}}\left(\left|u_{b_{n}}\right|+\left|u_{0}\right|\right)^{p-1}\left|u_{0}\right| d x \\
& \leq\left(\int_{\Omega_{R}^{C}}\left(\left|u_{b_{n}}\right|+\left|u_{0}\right|\right)^{2(p-1)} d x\right)^{\frac{1}{2}}\left(\int_{\Omega_{R}^{C}}\left|u_{0}\right|^{2} d x\right)^{\frac{1}{2}} \\
& \leq\left(\int_{\mathbb{R}^{3}}\left(\left|u_{b_{n}}\right|+\left|u_{0}\right|\right)^{2(p-1)} d x\right)^{\frac{1}{2}}\left(\int_{\Omega_{R}^{C}}\left|u_{0}\right|^{2} d x\right)^{\frac{1}{2}} \\
& \leq 4^{p-1}\left(\int_{\mathbb{R}^{3}}\left(\left|u_{b_{n}}\right|^{2(p-1)}+\left|u_{0}\right|^{2(p-1)}\right) d x\right)^{\frac{1}{2}}\left(\int_{\Omega_{R}^{C}}\left|u_{0}\right|^{2} d x\right)^{\frac{1}{2}} \\
& =4^{p-1}\left(\left|u_{b_{n}}\right|_{2(p-1)}^{2(p-1)}+\left|u_{0}\right|_{2(p-1)}^{2(p-1)}\right)^{\frac{1}{2}}\left(\int_{\Omega_{R}^{C}}\left|u_{0}\right|^{2} d x\right)^{\frac{1}{2}} \cdot
\end{aligned}
$$

Because of the boundedness of $\left\{u_{b_{n}}\right\}$ combined with (2.2), there exists $M_{1}>0$ such that

$$
4^{p-1}\left(\left|u_{n}\right|_{2(p-1)}^{2(p-1)}+\left|u_{0}\right|_{2(p-1)}^{2(p-1)}\right)^{\frac{1}{2}} \leq M_{1}
$$

On the other hand, the fact that $u_{0} \in L^{2}\left(\mathbb{R}^{3}\right)$ implies that $\forall \varepsilon>0$, we can choose $R>0$ large enough so that

$$
\left(\int_{\Omega_{R}^{C}}\left|u_{0}\right|^{2} d x\right)^{\frac{1}{2}}<\frac{\varepsilon}{2 M_{1}} .
$$

Then

$$
\left|\int_{\Omega_{R}^{C}}\left(\left|u_{b_{n}}\right|^{p-2}-\left|u_{0}\right|^{p-2}\right) u_{0}\left(u_{b_{n}}-u_{0}\right) d x\right|<\frac{\varepsilon}{2} .
$$

Similarly, for given $R$ above, there exists $M_{2}>0$ such that

$$
\begin{aligned}
B_{n} & :=\left|\int_{\Omega_{R}}\left(\left|u_{b_{n}}\right|^{p-2}-\left|u_{0}\right|^{p-2}\right) u_{0}\left(u_{b_{n}}-u_{0}\right) d x\right| \\
& \leq \int_{\Omega_{R}}\left(\left|u_{b_{n}}\right|^{p-2}+\left|u_{0}\right|^{p-2}\right)\left|u_{0}\right|\left|u_{b_{n}}-u_{0}\right| d x \\
& \leq \int_{\Omega_{R}}\left(\left|u_{b_{n}}\right|+\left|u_{0}\right|\right)^{p-2}\left|u_{0}\right|\left|u_{b_{n}}-u_{0}\right| d x \\
& \leq \int_{\Omega_{R}}\left(\left|u_{b_{n}}\right|+\left|u_{0}\right|\right)^{p-1}\left|u_{b_{n}}-u_{0}\right| d x \\
& \leq\left(\int_{\Omega_{R}}\left(\left|u_{b_{n}}\right|+\left|u_{0}\right|\right)^{2(p-1)} d x\right)^{\frac{1}{2}}\left(\int_{\Omega_{R}}\left|u_{b_{n}}-u_{0}\right|^{2} d x\right)^{\frac{1}{2}} \\
& \leq M_{2}\left(\int_{\Omega_{R}}\left|u_{b_{n}}-u_{0}\right|^{2} d x\right)^{\frac{1}{2}} .
\end{aligned}
$$


By the fact that $u_{b_{n}} \rightarrow u_{0}$ in $L_{\text {loc }}^{r}\left(\mathbb{R}^{3}\right), r \in[1,6)$, there exists $N_{1} \geq 1$, such that

$$
M_{2}\left(\int_{\Omega_{R}}\left|u_{b_{n}}-u_{0}\right|^{2} d x\right)^{\frac{1}{2}}<\frac{\varepsilon}{2}
$$

as $n \geq N_{1}$. Thus,

$$
\left|\int_{\Omega_{R}}\left(\left|u_{b_{n}}\right|^{p-2}-\left|u_{0}\right|^{p-2}\right) u_{0}\left(u_{b_{n}}-u_{0}\right) d x\right|<\frac{\varepsilon}{2}
$$

as $n \geq N_{1}$.

Hence, (3.8)-(3.9) shows that (3.7) holds.

Now, we show that

$$
\begin{aligned}
& \int_{\mathbb{R}^{3}} h(x)\left|u_{b_{n}}\right|^{q-2} u_{b_{n}}\left(u_{b_{n}}-u_{0}\right) d x \\
& \quad-\int_{\mathbb{R}^{3}} h(x)\left|u_{0}\right|^{q-2} u_{0}\left(u_{b_{n}}-u_{0}\right) d x \rightarrow 0, \quad \text { as } n \rightarrow \infty
\end{aligned}
$$

We only prove that $\int_{\mathbb{R}^{3}} h(x)\left|u_{b_{n}}\right|^{q-2} u_{b_{n}}\left(u_{b_{n}}-u_{0}\right) d x \rightarrow 0$ as $n \rightarrow \infty$, because the proof on $\int_{\mathbb{R}^{3}} h(x)\left|u_{0}\right|^{q-2} u_{0}\left(u_{b_{n}}-u_{0}\right) d x \rightarrow 0$ is similar.

We make an argument similar to (3.7) as follows:

For $R>0$, we have

$$
\begin{aligned}
& \left.\left|\int_{\mathbb{R}^{3}} h(x)\right| u_{b_{n}}\right|^{q-2} u_{b_{n}}\left(u_{b_{n}}-u_{0}\right) d x \mid \\
& \quad \leq \int_{\mathbb{R}^{3}} h(x)\left|u_{b_{n}}\right|^{q-1}\left|u_{b_{n}}-u_{0}\right| d x \\
& \quad=\int_{\Omega_{R}} h(x)\left|u_{b_{n}}\right|^{q-1}\left|u_{b_{n}}-u_{0}\right| d x+\int_{\Omega_{R}^{C}} h(x)\left|u_{b_{n}}\right|^{q-1}\left|u_{b_{n}}-u_{0}\right| d x
\end{aligned}
$$

Take $r_{1}=\frac{6}{q-1}, r_{2}=\frac{6}{1+\left(q_{1}-q\right)}$. Then the condition $(l)$ ensures that $1<r_{1}<2<r_{2}<6$. By the boundedness of $\left\{u_{b_{n}}\right\}$ combined with (2.2) and the fact that $u_{b_{n}} \rightarrow u_{0}$ in $L_{\text {loc }}^{r}\left(\mathbb{R}^{3}\right), r \in[1,6)$, there exists $M_{1}>0$ such that

$$
\begin{aligned}
A_{n} & :=\int_{\Omega_{R}} h(x)\left|u_{b_{n}}\right|^{q-1}\left|u_{b_{n}}-u_{0}\right| d x \\
& \leq\left(\int_{\Omega_{R}} h^{\frac{6}{6-q_{1}}} d x\right)^{\frac{6-q_{1}}{6}}\left(\int_{\Omega_{R}}\left|u_{b_{n}}\right|^{(q-1) r_{1}} d x\right)^{\frac{1}{r_{1}}}\left(\int_{\Omega_{R}}\left|u_{b_{n}}-u_{0}\right|^{r_{2}} d x\right)^{\frac{1}{r_{2}}} \\
& \leq|h|_{\frac{6}{6-q_{1}}}\left|u_{b_{n}}\right|_{6}^{q-1}\left(\int_{\Omega_{R}}\left|u_{b_{n}}-u_{0}\right|^{r_{2}} d x\right)^{\frac{1}{r_{2}}} \\
& \leq M_{1}\left(\int_{\Omega_{R}}\left|u_{b_{n}}-u_{0}\right|^{r_{2}} d x\right)^{\frac{1}{r_{2}}} \rightarrow 0
\end{aligned}
$$

as $n \rightarrow \infty$. 
Similarly, let $r=\frac{6 q}{q_{1}}, r_{1}=\frac{6}{q-1}, r_{2}=\frac{6}{1+\left(q_{1}-q\right)}$, then $4<r<6$ and there exists $M_{2}>0$ such that

$$
\begin{aligned}
B_{n}:= & \int_{\Omega_{R}^{C}} h(x)\left|u_{b_{n}}\right|^{q-1}\left|u_{b_{n}}-u_{0}\right| d x \\
\leq & \int_{\Omega_{R}^{C}} h(x)\left(\left|u_{b_{n}}\right|^{q}+\left|u_{b_{n}}\right|^{q-1}\left|u_{0}\right|\right) d x \\
\leq & \left(\int_{\Omega_{R}^{C}} h^{\frac{6}{6-q_{1}}} d x\right)^{\frac{6-q_{1}}{6}}\left(\int_{\Omega_{R}^{C}}\left|u_{b_{n}}\right|^{r} d x\right)^{\frac{q}{r}} \\
& +\left(\int_{\Omega_{R}^{C}} h^{\frac{6}{6-q_{1}}} d x\right)^{\frac{6-q_{1}}{6}}\left(\int_{\Omega_{R}^{C}}\left|u_{b_{n}}\right|^{r_{1}(q-1)} d x\right)^{\frac{1}{r_{1}}}\left(\int_{\Omega_{R}^{C}}\left|u_{0}\right|^{r_{2}} d x\right)^{\frac{1}{r_{2}}} \\
\leq & \left(\int_{\Omega_{R}^{C}} h^{\frac{6}{6-q_{1}}} d x\right)^{\frac{6-q_{1}}{6}}\left[\left|u_{b_{n}}\right|_{r}^{q}+\left|u_{b_{n}}\right|_{6}^{q-1}\left|u_{0}\right|^{r_{2}}\right] \leq M_{2}\left(\int_{\Omega_{R}^{C}} h^{\frac{6}{6-q_{1}}} d x\right)^{\frac{6-q_{1}}{6}} .
\end{aligned}
$$

Because $h \in L^{\frac{6}{6-q_{1}}}\left(\mathbb{R}^{3}\right)$, for any $\varepsilon>0$, we can choose $R>0$ large enough so that

$$
\left(\int_{\Omega_{R}^{C}} h^{\frac{6}{6-q_{1}}} d x\right)^{\frac{6-q_{1}}{6}}<\frac{\varepsilon}{M_{2}}
$$

and therefore

$$
\int_{\Omega_{R}^{C}} h(x)\left|u_{b_{n}}\right|^{q-1}\left|u_{b_{n}}-u_{0}\right| d x<\varepsilon
$$

Hence, by (3.11)-(3.12), we conclude that $\int_{\mathbb{R}^{3}} h(x)\left|u_{b_{n}}\right|^{q-2} u_{b_{n}}\left(u_{b_{n}}-u_{0}\right) d x \rightarrow 0$ as $n \rightarrow \infty$.

Finally, we show that

$$
\int_{\mathbb{R}^{3}} k(x)\left(\phi_{u_{b_{n}}}-\phi_{u_{0}}\right) u_{0}\left(u_{b_{n}}-u_{0}\right) d x \rightarrow 0 \quad \text { as } n \rightarrow \infty
$$

In fact, by Lemma 2.2(iii)-(iv) together with the boundedness of $\left\{u_{b_{n}}\right\}$ in $H^{1}\left(\mathbb{R}^{3}\right)$, we have

$$
\begin{aligned}
& \left|\int_{\mathbb{R}^{3}} k(x)\left(\phi_{u_{b_{n}}}-\phi_{u_{0}}\right) u_{0}\left(u_{b_{n}}-u_{0}\right) d x\right| \\
& \quad \leq \int_{\mathbb{R}^{3}} k(x)\left|\phi_{u_{b_{n}}}-\phi_{u_{0}}\right|\left|u_{0}\left(u_{b_{n}}-u_{0}\right)\right| d x \\
& \quad \leq|k|_{\infty}\left(\int_{\mathbb{R}^{3}}\left|\phi_{u_{b_{n}}}-\phi_{u_{0}}\right|^{6}\right)^{\frac{1}{6}}\left(\int_{\mathbb{R}^{3}}\left|u_{0}\left(u_{b_{n}}-u_{0}\right)\right|^{\frac{6}{5}} d x\right)^{\frac{5}{6}} \\
& \quad \leq|k|_{\infty}\left(\left|\phi_{u_{b_{n}}}\right|_{6}+\left|\phi_{u_{0}}\right|_{6}\right)\left(\int_{\mathbb{R}^{3}}\left|u_{0}\left(u_{b_{n}}-u_{0}\right)\right|^{\frac{6}{5}} d x\right)^{\frac{5}{6}} \\
& \quad \leq|k|_{\infty} \bar{S}^{-2} S_{6}^{-2}|k|_{2}\left(\left\|u_{b_{n}}\right\|^{2}+\left\|u_{0}\right\|^{2}\right)\left(\int_{\mathbb{R}^{3}} \mid u_{0}\left(u_{b_{n}}-u_{0}\right)^{\frac{6}{5}} d x\right)^{\frac{5}{6}} \\
& \quad \leq M_{1}\left(\int_{\mathbb{R}^{3}}\left|u_{0}\left(u_{b_{n}}-u_{0}\right)\right|^{\frac{6}{5}} d x\right)^{\frac{5}{6}} \cdot
\end{aligned}
$$


For any $R>0$, we know that

$$
\int_{\mathbb{R}^{3}}\left|u_{0}\left(u_{b_{n}}-u_{0}\right)\right|^{\frac{6}{5}} d x=\int_{\Omega_{R}}\left|u_{0}\left(u_{b_{n}}-u_{0}\right)\right|^{\frac{6}{5}} d x+\int_{\Omega_{R}^{C}}\left|u_{0}\left(u_{b_{n}}-u_{0}\right)\right|^{\frac{6}{5}} d x
$$

Because

$$
\begin{aligned}
\left(\int_{\Omega_{R}^{C}}\left|u_{0}\left(u_{b_{n}}-u_{0}\right)\right|^{\frac{6}{5}} d x\right) & \left.\leq\left.\left(\int_{\Omega_{R}^{C}}\left|u_{0}\right|^{\frac{3}{2}} d x\right)^{\frac{4}{5}}\left(\int_{\Omega_{R}^{C}} \mid u_{b_{n}}-u_{0}\right)\right|^{6} d x\right)^{\frac{1}{5}} \\
& \leq\left(\int_{\Omega_{R}^{C}}\left|u_{0}\right|^{\frac{3}{2}} d x\right)^{\frac{4}{5}}\left(\int_{\Omega_{R}^{C}}\left(\left|u_{b_{n}}\right|+\left|u_{0}\right|\right)^{6} d x\right)^{\frac{1}{5}} \\
& \leq\left(\int_{\Omega_{R}^{C}}\left|u_{0}\right|^{\frac{3}{2}} d x\right)^{\frac{4}{5}}\left(\left|u_{b_{n}}\right|_{6}+\left|u_{0}\right|_{6}\right)^{\frac{6}{5}} \\
& \leq M_{2}\left(\int_{\Omega_{R}^{C}}\left|u_{0}\right|^{\frac{3}{2}} d x\right)^{\frac{4}{5}}
\end{aligned}
$$

and $u_{0} \in L^{\frac{3}{2}}\left(\mathbb{R}^{3}\right)$, for any given $\varepsilon>0$, we can choose $R>0$ large enough so that

$$
M_{2}\left(\int_{\Omega_{R}^{C}}\left|u_{0}\right|^{\frac{3}{2}} d x\right)^{\frac{4}{5}}<\varepsilon / 2
$$

On the other hand, since $u_{b_{n}} \rightarrow u_{0}$ in $L_{\text {loc }}^{r}\left(\mathbb{R}^{3}\right), r \in[1,6)$, there exists $N>0$ such that

$$
\begin{aligned}
\int_{\Omega_{R}}\left|u_{0}\left(u_{b_{n}}-u_{0}\right)\right|^{\frac{6}{5}} d x & \left.=\left.\left(\int_{\Omega_{R}} \mid u_{b_{n}}-u_{0}\right)\right|^{\frac{3}{2}} d x\right)^{\frac{4}{5}}\left(\int_{\Omega_{R}}\left|u_{0}\right|^{6} d x\right)^{\frac{1}{5}} \\
& \left.\leq\left(\int_{\Omega_{R}} \mid u_{b_{n}}-u_{0}\right)^{\left.\right|^{\frac{3}{2}}} d x\right)^{\frac{4}{5}}\left(\int_{\mathbb{R}^{3}}\left|u_{0}\right|^{6} d x\right)^{\frac{1}{5}}<\varepsilon / 2
\end{aligned}
$$

as $n>N$. Thus, by (3.14)-(3.17), we find that (3.13) holds. Consequently, by (3.6), (3.7), (3.10) and (3.13), we obtain $d_{n} \rightarrow 0$ as $n \rightarrow \infty$, and therefore, $u_{b_{n}} \rightarrow u_{0}$ as $n \rightarrow \infty$ in $H^{1}\left(\mathbb{R}^{3}\right)$.

Now, by

$$
\begin{aligned}
\left\langle J_{0}^{\prime}\left(u_{0}\right), u_{b_{n}}^{ \pm}\right\rangle & =\left\langle J_{b_{n}}^{\prime}\left(u_{b_{n}}\right), u_{b_{n}}^{ \pm}\right\rangle-\left\langle J_{b_{n}}^{\prime}\left(u_{b_{n}}\right)-J_{0}^{\prime}\left(u_{b_{n}}\right), u_{b_{n}}^{ \pm}\right\rangle-\left\langle J_{0}^{\prime}\left(u_{b_{n}}\right)-J_{0}^{\prime}\left(u_{0}\right), u_{b_{n}}^{ \pm}\right\rangle \\
& =-\left\langle J_{b_{n}}^{\prime}\left(u_{b_{n}}\right)-J_{0}^{\prime}\left(u_{b_{n}}\right), u_{b_{n}}^{ \pm}\right\rangle-\left\langle J_{0}^{\prime}\left(u_{b_{n}}\right)-J_{0}^{\prime}\left(u_{0}\right), u_{b_{n}}^{ \pm}\right\rangle
\end{aligned}
$$

combined with the fact that $u_{b_{n}} \rightarrow u_{0}, u_{b_{n}}^{ \pm} \rightarrow u_{0}^{ \pm}$in $H^{1}\left(\mathbb{R}^{3}\right)$ and $b_{n} \rightarrow 0$ and that $J_{b}$ is a $C^{1}$ functional in $H^{1}\left(\mathbb{R}^{3}\right)$, we have $J_{0}^{\prime}\left(u_{0}\right)=0$ and

$$
\left\langle J_{0}^{\prime}\left(u_{0}\right), u_{0}^{ \pm}\right\rangle=\lim _{n \rightarrow \infty}\left\langle J_{0}^{\prime}\left(u_{0}\right), u_{b_{n}}^{ \pm}\right\rangle=0
$$

On the other hand, by an argument similar to (2.26) and a subsequent derivation on $u_{b}^{ \pm} \neq 0$, we know that $u_{0}^{ \pm} \neq 0$. So, $u_{0} \in M_{0}$. That is, $u_{0}$ is a sign-changing weak solution of problem (1.2) 
Now, we prove that $u_{0}$ is also a least energy sign-changing solution of problem (1.2). In fact, assume that $v_{0} \in M_{0}$ is a least energy sign-changing solution of problem (1.2). Then, by Lemma 2.4 , there is a sequence of pairs $\left(s_{b_{n}}, t_{b_{n}}\right) \in \mathbb{R}_{+}^{0} \times \mathbb{R}_{+}^{0}$ with $s_{b_{n}} v_{0}^{+}+t_{b_{n}} v_{0}^{-} \in M_{b_{n}}$. Because $b_{n} \rightarrow 0$ as $n \rightarrow \infty$, observing that (2.11) holds corresponding to $b=b_{n}, u=v_{0}$ and taking into account that $2 \leq p \leq 4<q<6, \lambda \geq 0$, and $h(x)>0$, a.e. $x \in \mathbb{R}^{3}$, it is easy to check that $\left\{\left(s_{b_{n}}, t_{b_{n}}\right)\right\}$ is bounded. Without loss of generality, we can assume that $s_{b_{n}} \rightarrow$ $s_{0} \in \mathbb{R}_{+}, t_{b_{n}} \rightarrow t_{0} \in \mathbb{R}_{+}$. Moreover, by an argument similar to that in (2)(ii) of the proof of Lemma 2.4, it can be verified that $s_{0}>0, t_{0}>0$. Consequently, we have

$$
s_{0} v_{0}^{+}+t_{0} v_{0}^{-}=\lim _{n \rightarrow \infty}\left(s_{b_{n}} v_{0}^{+}+t_{b_{n}} v_{0}^{-}\right) \in M_{0}
$$

Applying Lemma 2.4 for $b=0$, it follows from $v_{0} \in M_{0}$ that $s_{0}=t_{0}=1$. Thus, we have

$$
J_{0}\left(v_{0}\right) \leq J_{0}\left(u_{0}\right)=\lim _{n \rightarrow \infty} J_{b_{n}}\left(u_{b_{n}}\right) \leq \lim _{n \rightarrow \infty} J_{b_{n}}\left(s_{b_{n}} v_{0}^{+}+t_{b_{n}} v_{0}^{-}\right)=J_{0}\left(v_{0}^{+}+v_{0}^{-}\right)=J_{0}\left(v_{0}\right)
$$

Hence, $J_{0}\left(v_{0}\right)=J_{0}\left(u_{0}\right)$. That is, $u_{0}$ is also a least energy sign-changing solution of problem (1.2). The proof of Theorem 3.2 is complete.

\section{Conclusion}

In this paper, with the help of the constraint variational method combined with a quantitative lemma, Kirchhoff-Poisson systems (1.1) are investigated and the existence result on the least energy sign-changing solution with two nodal domains to the problem is established. Moreover, the convergence property of $u_{b}$ as $b \searrow 0$ is also obtained. It should be pointed out that, because the nonlocal terms $b \int_{\mathbb{R}^{3}}\left(|\nabla u|^{2} d x\right) \Delta u$ and $\phi_{u}$ are involved here, the above Kirchhoff-Poisson systems are totally different from the case $b=0$ and $k=0$ and there are more difficulties we need to overcome in the proof.

\section{Acknowledgements}

The authors wish to thank the anonymous referees for their valuable suggestions.

\section{Funding}

This work was supported by the National Natural Science Foundation of China (No. 11601139), the Natural Science Foundation of Hubei Province (No. 2019CFB522), and Scientific Research and Innovation Team of Hubei Normal University.

\section{Abbreviations}

Not applicable.

\section{Availability of data and materials}

Not applicable.

Competing interests

The authors declare that they have no competing interests.

Authors' contributions

All authors contributed equally to this paper. All authors read and approved the final manuscript.

\section{Publisher's Note}

Springer Nature remains neutral with regard to jurisdictional claims in published maps and institutional affiliations. 


\section{References}

1. Ambrosetti, A.: On Schrödinger-Poisson system. Milan J. Math. 76, 257-274 (2008)

2. Ambrosetti, A., Ruiz, D.: Multiple bound states for the Schrödinger-Poisson problem. Commun. Contemp. Math. 10, 391-404 (2008)

3. Azzollini, A., Pornponio, A.: Ground state solutions for the nonlinear Schrödinger-Maxwell equations. J. Math. Anal. Appl. 345, 90-108 (2008)

4. Cerami, G., Vaira, G.: Positive solutions for some non-autonomous Schrödinger-Poisson systems. J. Differ. Equ. 248, 521-543 (2010)

5. Zhong, X., Tang, C.: Ground state sign-changing solutions for a Schrödinger-Poisson system with a critical nonlinearity in $\mathbb{R}^{3}$. Nonlinear Anal., Real World Appl. 39, 166-184 (2018)

6. Batista, A.M., Furtado, M.F.: Positive and nodal solutions for a nonlinear Schrödinger-Poisson system with sign-changing potentials. Nonlinear Anal., Real World Appl. 39, 142-156 (2018)

7. Alves, C., Souto, M.: Existence of least energy nodal solution for a Schrödinger-Poisson system in bounded domains Z. Angew. Math. Phys. 65, 1153-1166 (2014)

8. Chen, S., Tang, X.: Ground state sign-changing solutions for a class of Schrödinger-Poisson type problems in $\mathbb{R}^{3}$. Z. Angew. Math. Phys. 67(4), 102 (2016)

9. Ianni, l.: Sign-changing radial solutions for the Schrödinger-Poisson-Slater problem. Topol. Methods Nonlinear Anal. 41, 365-385 (2013)

10. Liu, Z., Wang, Z., Zhang, J.: Infinitely many sign-changing solutions for the nonlinear Schrödinger-Poisson system. Ann. Math. 195, 775-794 (2016)

11. Chen, J.: Multiple positive solutions of a class of non autonomous Schrödinger-Poisson systems. Nonlinear Anal., Real World Appl. 21, 13-26 (2015)

12. Ianni, I., Vaira, G.: Non-radial sign-changing solutions for the Schrödinger-Poisson problem in the semiclassical limit. NoDEA Nonlinear Differ. Equ. Appl. 22, 741-776 (2015)

13. Alves, C., Souto, M., Soares, S.: A sign-changing solution for the Schrödinger-Poisson equation in $\mathbb{R}^{3}$. Rocky Mt. J. Math. 47, 1-25 (2017)

14. Sun, J., Wu, T:: On the nonlinear Schrödinger-Poisson systems with sign-changing potential. Z. Angew. Math. Phys. 66, 1649-1669 (2015)

15. Figueiredo, G.: Existence of a positive solution for a Kirchhoff problem type with critical growth via truncation argument. J. Math. Anal. Appl. 401, 706-713 (2013)

16. Li, Y., Li, F., Shi, J.: Existence of a positive solution to Kirchhoff type problems without compactness conditions. J. Differ. Equ. 253, 2285-2294 (2012)

17. Naimen, D.: The critical problem of Kirchhoff type elliptic equations in dimension four. J. Differ. Equ. 257, 1168-1193 (2014)

18. Naimen, D.: Positive solution of Kirchhoff type elliptic equations involving a critical Sobolev exponent. NoDEA Nonlinear Differ. Equ. Appl. 21, 885-914 (2014)

19. Sun, J., Tang, C.: Existence and multiplicity of solutions for Kirchhoff type equations. Nonlinear Anal. 74, 1212-1222 (2011)

20. Wu, Y., Liu, S.: Existence and multiplicity of solutions for asymptotically linear Schrödinger-Kirchhoff equations. Nonlinear Anal., Real World Appl. 26, 191-198 (2015)

21. Zhang, D., Chai, G., Liu, W.: Existence and concentration of solutions for the nonlinear Kirchhoff type equations with steep well potential. Bound. Value Probl. 2017, 142 (2017)

22. Baraket, S., Molica Bisci, G.: Multiplicity results for elliptic Kirchhoff-type problems. Adv. Nonlinear Anal. 6(1), 85-93 (2017)

23. $\mathrm{Xu}, \mathrm{L} ., \mathrm{Chen}, \mathrm{H}$.: Ground state solutions for Kirchhoff-type equations with a general nonlinearity in the critical growth. Adv. Nonlinear Anal. 7(4), 535-546 (2018)

24. Jeanjean, L.: On the existence of bounded Palais-Smale sequences and application to a Landesman-Lazer-type problem set on $\mathbb{R}^{N}$. Proc. R. Soc. Edinb., Sect. A 129, 787-809 (1999)

25. Molica Bisci, G., Rădulescu, V.D.: Ground state solutions of scalar field fractional Schrödinger equations. Calc. Var. Partial Differ. Equ. 54(3), 2985-3008 (2015)

26. Xiang, M., Rădulescu, V.D., Zhang, B.: Fractional Kirchhoff problems with critical Trudinger-Moser nonlinearity. Calc. Var. Partial Differ. Equ. 58(2), 57 (2019)

27. Molica Bisci, G., Radulescu, V.D., Servadei, R.: Variational Methods for Nonlocal Fractional Problems. Encyclopedia of Mathematics and Its Applications, vol. 162. Cambridge University Press, Cambridge (2016)

28. Shuai, W.: Sign-changing solutions for a class of Kirchhoff-type problem in bounded domains. J. Differ. Equ. 259, 1256-1274 (2015)

29. Tang, X., Cheng, B.: Ground state sign-changing solutions for Kirchhoff type problems in bounded domains. J. Differ. Equ. 261, 2384-2402 (2016)

30. Zhang, Q:: Multiple positive solutions for Kirchhoff-Schrödinger-Poisson system with general singularity. Bound. Value Probl. 2017, 127 (2017)

31. Liu, F., Wang, S.: Positive solutions of Schrödinger-Kirchhoff-Poisson system without compact condition. Bound. Value Probl. 2017, 156 (2017)

32. Wang, L., Rădulescu, V.D., Zhang, B.: Infinitely many solutions for fractional Kirchhoff-Schrödinger-Poisson systems. J. Math. Phys. 60, 011506 (2019). https://doi.org/10.1063/1.5019677

33. Willem, M.: Minimax Theorems. Birkhäuser, Basel (1996) 